Purdue University

Purdue e-Pubs

2002

\title{
An Experimental and Numerical Investigation of the Bridgman Growth of a Transparent Material
}

JE. Simpson

S V. Garimella

Indiana University Purdue University Indianapolis, sureshg@purdue.edu

H C. de Groh III

Follow this and additional works at: http://docs.lib.purdue.edu/coolingpubs

Simpson, J E.; Garimella, S V.; and de Groh III, H C., "An Experimental and Numerical Investigation of the Bridgman Growth of a Transparent Material" (2002). CTRC Research Publications. Paper 298.

http://docs.lib.purdue.edu/coolingpubs/298

This document has been made available through Purdue e-Pubs, a service of the Purdue University Libraries. Please contact epubs@purdue.edu for additional information. 


\title{
An Experimental and Numerical Investigation of the Bridgman Growth of a Transparent Material ${ }^{\text {TI }}$
}

\author{
James E. Simpson ${ }^{\S}$ and Suresh V. Garimella ${ }^{\dagger}$ \\ School of Mechanical Engineering \\ Purdue University \\ West Lafayette, Indiana, 47907-1288 USA \\ Phone: (765) 494-5621 Fax: (765) 494-0539 \\ Email: sureshg@ecn.purdue.edu \\ Henry C. de Groh III \\ National Aeronautics and Space Administration \\ Mail Stop 105-1, Glenn Research Center \\ Cleveland, Ohio, 44135 USA
}

\begin{abstract}
A combined experimental and numerical study of the horizontal Bridgman growth of pure succinonitrile $(\mathrm{SCN})$ has been performed. The effect of convection on interface propagation and shape is quantified and discussed. Measurements were obtained both under conditions of nogrowth and for a $40 \mu \mathrm{m} / \mathrm{s}$ growth rate. The quantities measured include interface shape and location, melt velocities, and temperature boundary conditions on the ampoule exterior. The melt velocities were measured using a new technique that employed digital cameras to image the locations of seed particles in the melt. The growth front was stable and non-dendritic, but was significantly distorted by the influence of convection in the melt and, for the growth case, by the moving temperature boundary conditions along the ampoule. Both two- and three-dimensional numerical simulations of the growth process were performed. Temperatures throughout the phase change material and ampoule as well as melt velocities were obtained from the simulations. The predicted interface shapes and melt velocities agree well with experimental results. Two different numerical algorithms were used; the utility of each for simulating phase-change problems is discussed. This combined experimental and numerical study provides a database for the validation of phase-change numerical models, in addition to furnishing detailed information about the influence of convection on the Bridgman growth process. In ongoing work, the computer models presented in this study are being used to simulate alloy solidification problems.
\end{abstract}

\footnotetext{
${ }^{\text {II }}$ Submitted for publication in AIAA Journal of Thermophysics and Heat Transfer, November 2000, and in revised form, January 2002.

${ }^{\S}$ Postdoctoral Research Associate

${ }^{\dagger}$ Associate Professor, person to whom correspondence should be addressed

ॠ Materials Engineer, Microgravity Science Division
} 


\section{NOMENCLATURE}

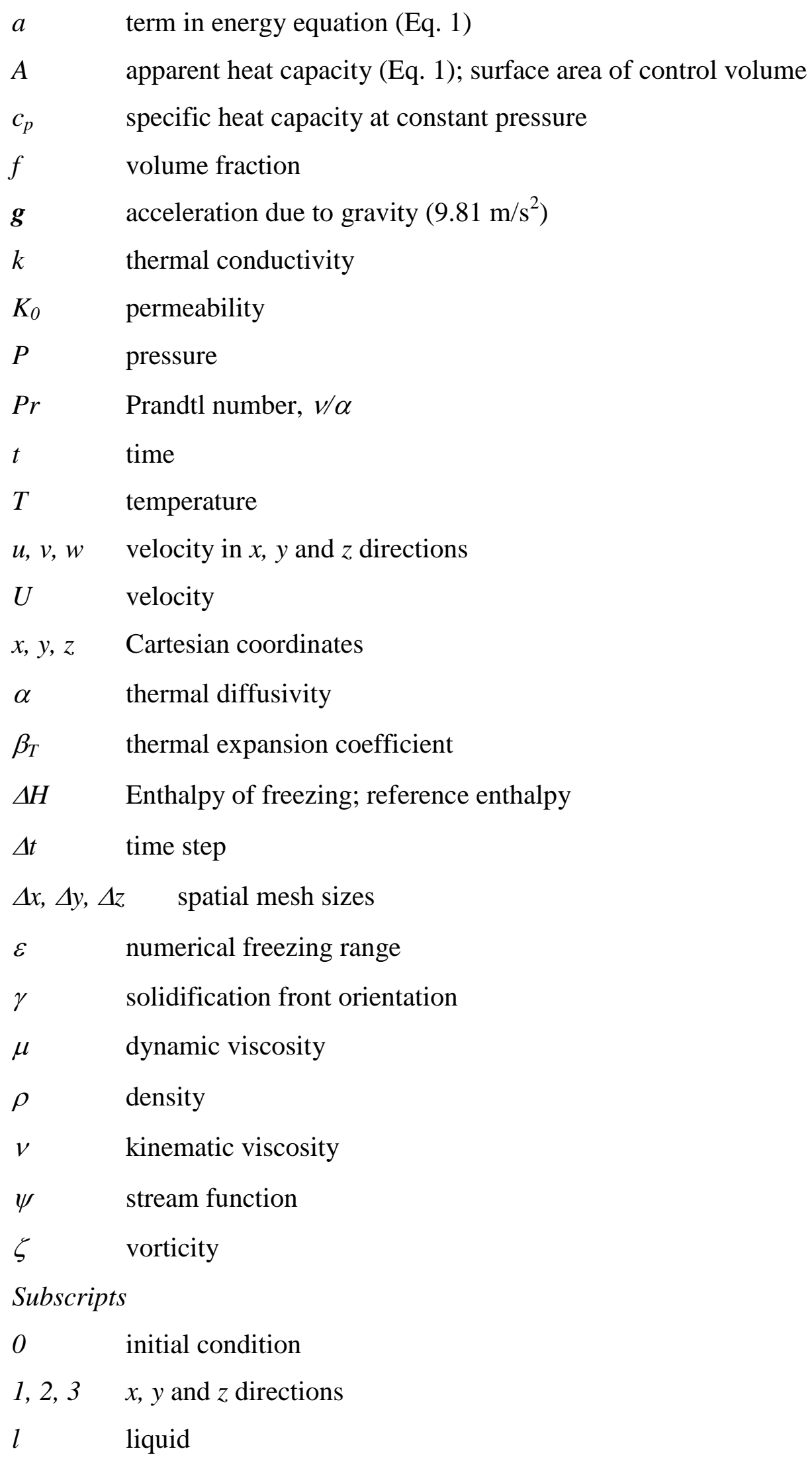


$m \quad$ at solidification front

Superscripts

$\wedge \quad$ unit vector

$\sim \quad$ vector

* $\quad$ at solidification front

$n \quad$ time step $\mathrm{n}$

\section{INTRODUCTION}

Increasingly, advanced materials used in the aerospace, automotive, optical and electronic fields require low levels of defects and high levels of solute uniformity. Directional solidification by the Bridgman process is widely used for synthesis of these high-quality materials. During Bridgman crystal growth, heat and mass transfer by both diffusion and convection driven by thermal and solutal gradients influence the shape of the solid/liquid interface and dopant segregation levels, thus directly determining the final crystal quality ${ }^{1}$. Key process parameters include the applied furnace temperature distribution and rate of translation, ampoule properties and furnace orientation.

Experimental investigations of solidification processes which involve metallic melts are complicated by the opacity, reactivity and high temperatures of the melts. Accurate experimental determination of the interface shape and convection is difficult. As a result, solidification experiments have often been performed with transparent materials that solidify in a manner analogous to metals ${ }^{2-9}$. Computer simulations have also been employed to investigate solidification processes. ${ }^{10-16}$ Such computer models are validated and enhanced by comparison with experimental results whenever these are available.

The present study has four main aims. First, a series of carefully designed experiments of the Bridgman solidification of pure succinonitrile (SCN) were performed. Interface shapes and positions were measured using charge-coupled device (CCD) camera images of the interface. 
Temperatures applied to the exterior of the ampoule by the heating and cooling jackets were also measured. The database of temperatures and interface shapes thus obtained is intended for use in validating numerical models of solidification. Second, the lack of experimental velocity measurements in the crystal growth literature was addressed by quantifying convective velocities in the melt region. This was achieved by reconstructing digital images of seed particles in the melt and hence determining the melt velocity field. Third, two numerical models of phasechange with convection were developed. These models were then validated by a comparison between the numerical results and the experimental data for interface shapes and melt velocities. Results from these two different numerical solution schemes were compared. It was found that a primitive variables formulation is preferable; this scheme is therefore being employed in ongoing work in alloy solidification. Finally, the results from our combined experimental-numerical database were used to gain valuable insight into the role of convection in the Bridgman solidification process.

\section{EXPERIMENTAL SETUP}

The experiments were conducted in the Transparent Directional Solidification Furnace (TDSF), located at the NASA Glenn Research Center, which is shown schematically in Fig. 1. Photographs of the facility are shown in Fig. 2. The TDSF is a Bridgman-type furnace with two copper jackets separated by an air gap which acts as the gradient or adiabatic zone between the two furnaces and also permits viewing of the glass ampoule which contains the phase change material. Constant-temperature circulating water baths provide heating or cooling for the jackets. The apparatus may be oriented in any direction; however, only the horizontal configuration is considered in this work. 
The $150 \mathrm{~mm}$ long ampoules used are made of borosilicate glass with a square cross section. The ampoules have an outside dimension of $8 \mathrm{~mm}$ and have a wall thickness of approximately 1 mm (Fig. 3). The thermal jackets have a square hole $11 \mathrm{~mm}$ on the side running through their entire lengths into which the (smaller) ampoule is inserted. The thermal field imposed on the ampoule is complicated due to convection and conduction in the air surrounding the ampoule, and so, accurate alignment and centering of the ampoule within the jackets is essential to obtaining repeatable results. For growth experiments, a servomotor with reducing gear translates the cooling jackets at a constant velocity of $40 \mu \mathrm{m} / \mathrm{s}$. The motor and indexer are also capable of positioning the jackets very accurately.

Ampoules containing pure SCN were used in this study. The ampoules were filled and sealed under vacuum conditions. The SCN was first purified to eliminate any spurious solutal convection. The purification process involved distillation and zone-refining in a hermetically sealed glass unit. ${ }^{17}$ Porous $\mathrm{SiO}_{2}$ seed particles $(63-90 \mu \mathrm{m}$ in diameter) were then added for use in resolving the velocities in the melt.

\section{Temperature Measurements}

For measuring the thermal boundary conditions on the outside of the ampoule, K-type (chromel-alumel) thermocouples were glued to the ampoule exterior using an epoxy. The instrumented ampoule used in previous work ${ }^{2}$ was employed, for which the general arrangement of the five thermocouples is as shown in Fig. 3. These were placed mid-way between the bottom corners at $(0,-4 \mathrm{~mm})$, referred to as the bottom middle (BM) thermocouple, between the top corners at $(0,4 \mathrm{~mm})$ called the top middle $(\mathrm{TM})$ thermocouple, on the rear side wall $(\mathrm{R})$ at $(-4$ $\mathrm{mm}, 0)$ and on the bottom and top corners (BC and TC) at $(-4 \mathrm{~mm},-4 \mathrm{~mm})$ and $(4 \mathrm{~mm}, 4 \mathrm{~mm})$, respectively. The thermocouple signals were recorded on a personal computer via a data acquisition system. 
For the temperature measurements, data were obtained as follows. The starting position of the edge of the cold jacket was noted using the position encoder on the servomotor. The jacket was then translated until the edge of the cold furnace was in the same position as each thermocouple. In this way the locations of each thermocouple (relative to the start position) were found. For measurements taken under growth conditions, temperature readings were made at intervals of time corresponding to the jackets translating $1 \mathrm{~mm}$ (i.e. to take temperatures every 1 $\mathrm{mm}$ during the $40 \mu \mathrm{m} / \mathrm{s}$ growth case, temperatures were recorded every 25 seconds). A total of more than 40 measurements were obtained for each thermocouple for a given experiment. The procedure for measurements under no-growth conditions was more complicated. Since the interface is stationary for this case, and the thermocouples fixed to the outside of the ampoule, a series of steady-state measurements were obtained with the interface formed at different locations along the ampoule as follows. Initially, the jackets were translated to a position where most of the ampoule was under the hot jacket. As a result, the majority of the SCN was liquid, and the interface formed closest to one end of the ampoule. Equilibrium was established after about 15 minutes, and the temperatures read. The motor was then used to move the jackets by an increment of $1 \mathrm{~mm}$, such that $1 \mathrm{~mm}$ more of the cold jacket covered the ampoule. Again, the apparatus was allowed to reach steady state and temperature measurements were obtained. By repeating this procedure, the entire temperature profile - relative to the interface location - was obtained.

The experimental error involved in locating the thermocouples is estimated to be $\pm 0.3 \mathrm{~mm}$. This error along with the finite thermal conductivity in the epoxies used to bond the thermocouples to the outside of the glass ampoule and inherent thermocouple variability result in an estimated uncertainty of $\pm 1^{\circ} \mathrm{C}$ in the temperature readings. 


\section{Interface Shape Measurements}

Interface shapes were determined by analyzing images of the interface captured using CCD video cameras mounted to capture footage of the interface from the top and side of the ampoule (refer to Figs. 1 and 2). Sample interface images shown in Fig. 4 indicate that the interface is sharp and non-dendritic even though it has been distorted and curved by convection. In the top view shown in Fig. 4(a), the interface takes on a symmetric crescent shape in the plane of maximum deflection which occurs in the mid horizontal plane or MHP and the plane where the interface meets the top wall of the ampoule (THP or top horizontal plane). The interface meets the bottom wall (BHP or bottom horizontal plane) at a very acute angle. The shape of the interface at the bottom is barely discernible in Fig. 4(a). In the side view shown in Fig. 4(b), the interface deflects the most along the centerline of the ampoule also referred to as the mid vertical plane or MVP. The location of the interface in the MVP is the right-most edge of the dark gray crescent. The location of the interface where it meets the front wall of the ampoule (the front vertical plane or FVP) can also be seen in this view.

The interface is only measured in these planes, where it can be clearly delineated. The planes are parallel to the observer; light from the measurement plane to the observer therefore travels in a perpendicular path. Consequently, there is no distortion of the light (and therefore no additional measurement error) as it passes through the succinonitrile, through the glass, and into ambient air, although these three media have different refractive indices.

The captured images were examined using image-processing software. In some cases, rudimentary image filtering was employed to enhance the edges of the interface. The experimental error in interface locations is estimated to be $\pm 0.2 \mathrm{~mm}$. 


\section{Velocity Measurements}

The motion of seed particles in the melt was filmed for a period of $10 \mathrm{~min}$. for the no-growth case. For the growth cases, particles near the interface were observed and filmed by sliding the cold furnace away from the interface zone to reveal the interface. This was done for maximum periods of $40 \mathrm{~s}$ in order to minimize the impact of the changing thermal boundary conditions. The interface was observed to continue at the normal growth rate for this length of time. If the cold zone was removed for any longer the interface movement began to slow down noticeably, indicating that the thermal conditions had appreciably changed.

A novel lighting condition was employed to reveal the particles while minimizing glare from surfaces and scratches in the ampoule. This involved pointing two focused beams from fiberoptic light sources equipped with polarizing filters along the axis of the ampoule, one directed from outside the hot zone towards the cold zone and the other acting in the opposite sense. Lighting conditions are crucial for the acquisition of acceptable video images of the seed particles; no other positions for the light sources yielded acceptable results. The number density of particles in the melt was high, making flow visualization straightforward.

The following calibration procedure determined the magnification of the monitor image, enabling measurements of the flow velocities from the video. The coordinate system was drawn on each monitor midway between the copper jackets and along the longitudinal centerline of the ampoule (typical monitor images are shown in Fig. 5). It is estimated that the $x, z$ and $y, z$ origins were found to within $\pm 0.13 \mathrm{~mm}$. The accuracy of determining the magnification is estimated to be within $\pm 1.5 \%$, and the location uncertainty was $\pm 0.03 \mathrm{~mm}$. The impact of this location uncertainty is dependent on the velocity and can be very large at small velocities, since the distance traveled is small but the error $( \pm 0.03 \mathrm{~mm})$ stays the same. Since velocities were measured at approximately $0.5 \mathrm{~s}$ intervals, the location uncertainty can be translated directly into 
a velocity uncertainty of $\pm 0.06 \mathrm{~mm} / \mathrm{s}$. Stokes' settling rate for the seed particles was estimated to be $0.0025 \mathrm{~mm} / \mathrm{s}$, compared with a typical value of $\sim 1 \mathrm{~mm} / \mathrm{s}$ for the measured velocities so that the difference between the particle velocity and the actual flow velocity is considered negligible. Based on this discussion, the composite uncertainty in the velocity measurements is judged to be no more than $\pm 0.08 \mathrm{~mm} / \mathrm{s}$. The fully automated version of the procedure described above is known as Stereoscopic Imaging Velocimetry (SIV) ${ }^{18}$. This new approach to obtaining threedimensional velocity measurements in fluids is being used by researchers at NASA Glenn in ongoing work.. Further details on the experimental procedure for measuring melt velocities may be found in Simpson et al. ${ }^{18}$

\section{EXPERIMENTAL RESULTS}

\section{Temperature Data}

Temperature measurements on the outside of the ampoule for solidification at a growth rate of $40 \mu \mathrm{m} / \mathrm{s}$ are shown in Fig. 6 . The cold and hot zone water bath temperature set points were 12 and $75^{\circ} \mathrm{C}$ respectively, with an adiabatic zone length of $5 \mathrm{~mm}$. Figure 6 contains data averaged from two different experiments; the values in these experiments agreed to within $\pm 1^{\circ} \mathrm{C}$. The top middle (TM) and top corner (TC) thermocouples record the highest temperatures, while the bottom middle $(\mathrm{BM})$ and bottom corner $(\mathrm{BC})$ record the lowest. This is in agreement with the observed interface shapes (refer to Fig. 4); solid is melted away from the top half of the domain indicating higher temperatures at the top of the ampoule. Once the material is completely solid $(z<-7 \mathrm{~mm})$, convection in the ampoule ceases and the temperature traces trend to a single line. The values obtained in the current experiment agree reasonably well with those obtained by de Groh and Lindstrom ${ }^{2}$, to within experimental uncertainty. For the numerical modeling under no- 
growth conditions presented in this study, the temperature measurements from previous work ${ }^{2}$ (which employed the same apparatus) were used.

\section{Interface Shape Data}

Interface shapes under no-growth conditions are shown in Fig. 7. Figure 7(a) is a plot of the front shapes in $x-z$ (horizontal) planes: MHP, BHP and THP are respectively the horizontal planes through the height at which maximum interface deflection occurs $(x,-1.6, z)$, and along the inside bottom and top ampoule walls $(x,-3, z)$ and $(x, 3, z)$. The interface shapes in the MHP and THP are crescent-shaped, with the solid side being concave. The slight asymmetry in the results for MHP may be due to a slight misalignment in the ampoule. The interface at the BHP meets the bottom wall at an acute angle making the contact line difficult to distinguish. Under ideal conditions this line should be symmetric. However the shape of this interface is very sensitive to thermal conditions, and very small misalignments can lead to asymmetries such as that in the figure.

Figure 7(b) details the front shapes in $y-z$ (vertical) planes: MVP and FVP refer to the vertical planes running along the longitudinal centerline $(x=0)$ and the inside front wall of the ampoule $(x=3)$. The interface shape at the FVP should be identical to that at the rear vertical plane $(x=$ -3) from symmetry. Note that the interface is considerably distorted from the vertical, with the solid forming a concave shape. The maximum deflection of the interface occurs along the MVP. The interface in this plane extends from a position of $(0,-3,2.09)$ to $(0,1.46,-2.00)$ - a total displacement of $4.09 \mathrm{~mm}$. The FVP is not as severely deflected from the vertical. The distorted interface shapes indicate that convection in the melt region has a significant impact on thermal transport in the domain.

Interface locations for the $40 \mu \mathrm{m} / \mathrm{s}$ growth case are shown in Fig. 8. The interface shape in the horizontal mid plane $(y=1.5)$ is crescent shaped as in the no-growth case but is much more 
concave. Interface locations in the other horizontal planes could not be determined quantitatively due to inadequate clarity of the video images. Interface shapes in the MVP and FVP show that compared to the no-growth case, the interface has become much more elongated but has retained the same general shape. The maximum total deflection has increased to 7.09 $\mathrm{mm}$, compared with $4.09 \mathrm{~mm}$ for the no-growth case. The separation between the interfaces in the FVP and MVP is also larger than for the no-growth case, due to the imposition of translating boundary conditions.

For Bridgman growth conditions, radial temperature gradients often exist in the adiabatic zone. Near the hot furnace, the sides of the sample are warmer than the center, and conversely near the cold furnace. These radial temperature gradients due to the applied thermal condition may influence the interface shape. Under growth conditions additional radial thermal gradients are produced since the temperatures in the sample lag behind those of the (translating) furnace due to the finite thermal conductivity of the sample. For the Bridgman growth of metals under conduction-dominated conditions, these radial gradients result in an interface shape that is

slightly concave or convex. ${ }^{10}$ For the cases shown in this work, horizontal Bridgman growth is considered. Observations made for SCN using the same apparatus as in this work but in the vertical Bridgman configuration (minimizing convection) reveal that the interface is flat during no-growth. ${ }^{19}$ Thus the concavity and asymmetry of the interface shapes in Fig. 7 during nogrowth is due to natural convection. The interface concavity and asymmetry in the growth case (Fig. 8), however, is caused by both convection and conduction effects.

\section{Velocity Data}

The following observations can be made about the flow patterns visualized in both the nogrowth and growth cases. Footage of the ampoule from the side (i.e. images of the $y$ - $z$ plane) reveals a single, longitudinal, primary convective cell. The flow moves along the top wall 
towards the interface; as it approaches the (cold) interface it is forced down and away from the interface in an arc shaped in a "reverse-C". The paths that particles travel near the interface resemble the shape of the interface itself. The fluid returns to the hot melt region by traveling along the bottom wall away from the interface. Particles that travel near the interface are clearly affected by the viscous layer near the wall and are noticeably slower. Some particles were observed to travel very near the interface and drift very slowly. Occasionally, these particles would become entrained in the approaching front for the growth case. Observations of the ampoule from above (i.e. images of the $x-z$ plane) reveal the secondary flows present near the interface. Particles that travel toward the interface along the centerline of the ampoule are forced downward as they approach the interface and return towards the hot zone along the centerline (i.e. zero movement in the $x$ direction). A particle that is adjacent to the centerline is forced outward from the centerline as it nears the interface. Such a particle then slides down the interface (thus the flow is clearly influenced by the shape of the interface) and returns to the bulk. These observations indicate that the convective flow is primarily a single two-dimensional cell in the $y-z$ plane with secondary convective motions in $x$ directions near the interface. Some similar flow structure features to those described here have been reported elsewhere. ${ }^{20}$

Measured velocities during the no-growth case are shown in Fig. 9. Locations and velocity values for three particles (denoted particle \#8, \#12 and \#13) are shown. The motion of the fluid particles shown in Fig. 9 illustrates the qualitative description provided above. The maximum measured value for velocity was found to be $1.50 \pm 0.08 \mathrm{~mm} / \mathrm{s}$. The location for this maximum was close to the centerline and near the interface at $(0.068,-0.3625,-0.567)$. Note that these relatively small velocity values are sufficient to cause the concave, three-dimensional interface shape shown in Fig. 7. The total interface deflection for the no-growth case is approximately 4 $\mathrm{mm}$, and is entirely due to these convective velocities. For the $40 \mu \mathrm{m} / \mathrm{s}$ growth case, the 
deflection increases to $8 \mathrm{~mm}$ due to transient conduction effects induced by the moving thermal jackets in addition to convection.

\section{MATHEMATICAL MODEL AND NUMERICAL ANALYSIS}

\section{Governing Equations}

The governing equation for energy transport with phase change, following the temperaturetransforming method of Zeng and Faghri ${ }^{13}$ is:

$$
\begin{aligned}
& A(T) \frac{\partial T}{\partial t}+\tilde{\nabla} \cdot\left[\rho \tilde{U} c_{p l} T\right]=\tilde{\nabla} \cdot(k \tilde{\nabla} T) \\
& A(T)=\rho c_{p}+a(T) \frac{\partial f_{l}}{\partial T} \\
& a(T)=\rho\left[\left(c_{p l}-c_{p s}\right) T+\Delta H\right]
\end{aligned}
$$

In Eq. (1) $A$ is termed an apparent or effective heat capacity, ${ }^{21}$ and accounts for the release of latent heat as solid is formed. Densities in the liquid and solid are assumed to be constant and equal. The governing equation for mass conservation for incompressible flow is the continuity equation:

$$
\tilde{\nabla} \cdot \tilde{U}=0
$$

The governing equations for momentum conservation are the Navier-Stokes equations:

$$
\frac{\partial \tilde{U}}{\partial t}+\tilde{U} \cdot \tilde{\nabla} \tilde{U}=-\frac{\tilde{\nabla} P}{\rho}+v \nabla^{2} \tilde{U}+\frac{K_{0}}{\rho} \frac{\left(1-f_{l}^{2}\right)}{f_{l}^{3}} \tilde{U}-\tilde{g} \beta_{T}\left(T-T_{0}\right)
$$

The RHS of Eq. (3) contains two source terms. The first is a momentum sink term that is used to extinguish velocities in the solid. ${ }^{22}$ The effect of this momentum sink is to immobilize the material in the fully solid regime $\left(f_{l}=0\right)$, while having no effect in the fully liquid regime $\left(f_{l}=\right.$ 1). An alternative method of immobilizing the solid would be to use a variable viscosity function. $^{21}$ The second source term is the Boussinesq approximation for the creation of momentum from density changes due to thermal gradients. Equations (2) and (3) are known as 
the pressure-velocity or primitive variable formulation of the governing equations for velocity. The solution of these equations, coupled with the energy field solution from Eq. (1), yields the velocity field in the liquid due to thermal convection.

An alternative formulation of the governing equations for the velocity field is also employed in this work. It can be shown that pressure can be eliminated from the equation set by defining two new variables, vorticity $(\tilde{\zeta})$ and vector potential $(\tilde{\psi})$. The most rigorous derivation of this approach is that of Hirasaki and Helums. ${ }^{23}$ The equations derived are the vorticity transport equation:

$$
\frac{\partial \tilde{\zeta}}{\partial t}+\tilde{U} \cdot(\tilde{\nabla} \tilde{\zeta})=v \nabla^{2} \tilde{\zeta}-\beta_{T}(\tilde{\nabla} \times T \tilde{g})
$$

and the vector potential equation:

$$
\nabla^{2} \tilde{\psi}=-\tilde{\zeta}
$$

Velocities are given by:

$$
\tilde{U}=\tilde{\nabla} \times \tilde{\psi}
$$

On an arbitrarily aligned three-dimensional surface, the boundary conditions on Eq. (4) become very difficult to handle. For this reason, and also for increased solution efficiency, the pressure-velocity formulation is used for 3D problems in the present study. The vorticity-vector potential approach is used only for $2 \mathrm{D}$ problems, where the boundary conditions at the interface are tractable. In two dimensions, Eqs. (4-6) reduce to:

$$
\begin{aligned}
& \frac{\partial \zeta_{3}}{\partial t}+u \frac{\partial \zeta_{3}}{\partial x}+v \frac{\partial \zeta_{3}}{\partial y}=v\left(\frac{\partial^{2} \zeta_{3}}{\partial x^{2}}+\frac{\partial^{2} \zeta_{3}}{\partial y^{2}}\right)-g \beta_{T} \frac{\partial T}{\partial x}-g \beta_{C} \frac{\partial C}{\partial x} \\
& \nabla^{2} \psi_{3}=-\zeta_{3} \\
& u=-\frac{\partial \psi_{3}}{\partial y}, v=\frac{\partial \psi_{3}}{\partial x}
\end{aligned}
$$


The $z$-component of the vector potential - a scalar - is the stream function for the $2 \mathrm{D}$ flow in the $x$-y plane. ${ }^{24}$ Lines of equivalent stream function are streamlines for the flow. Solving Eqs. (7) and (8) subject to the appropriate boundary and initial conditions constitutes the vorticitystream function or derived variable formulation for the velocity field in the melt. One of the advantages of this approach is that mass is guaranteed to be conserved. ${ }^{25}$ Unlike for the pressure-velocity formulation, the boundary conditions must be applied on the solid/liquid interface, and the solution domain is decomposed into the liquid and solid regions for the purposes of calculating velocities. This is achieved by reconstructing the location and shape of the solid/liquid front a posteriori during the solution of the governing equations. A comparison of the results obtained using both solution schemes is given in the next section, and is one of the contributions of this work.

\section{Numerical Schemes}

Two different solution schemes were employed. Scheme I used the derived variable $(\zeta-$ $\psi)$ approach for velocities, while Scheme II employed the primitive variable $(P-U)$ formulation. The methodology used for the energy equation was identical for both Scheme I and II. Scheme I is described in detail in Simpson and Garimella; ${ }^{12}$ salient details are provided here. A regular, finite-volume grid is used to solve the energy equation (Eq. 1), while a regular, finite-difference grid is used to solve the vorticity transport (Eq. 7) and stream function (Eq. 8) equations. The scheme is two-dimensional in space and fully transient. At time step $n$ the field variables are known, as is the front location and orientation. The algorithm marches to time step $n+1$ via the following steps:

- The energy equation (1) is solved using a sequential solution algorithm. Either SOR or MSI iteration is used to solve the discretized equations. UDS interpolation is used for convective fluxes, and deferred correction is used in the source terms for both equations. Velocity values 
from the previous time step $(n)$, interpolated from the finite mesh point locations to the finite volume face centers, are used. Once convergence is achieved, the solution vectors at $n+l$ for temperature $\left(T^{n+1}\right)$ and liquid fraction $\left(f_{l}^{n+1}\right)$ are known. A finite but small freezing range is required for numerical stability. This range was taken to be $0.5^{\circ} \mathrm{C}$, which approximately corresponds to the change in temperature between adjacent mesh points near the solid/liquid front.

- Next, the front location and orientation are reconstructed using the procedure of Hirt and Nichols, ${ }^{26}$ yielding values for the front location and orientation $\left(\gamma^{n+1}\right)$ at the new time step.

- The vorticity transport equation (7) is solved directly using Samarskii-Andreyev ADI factorization. ${ }^{27}$ This yields the vorticity values at time step $n+1\left(\zeta^{n+1}\right)$.

- The Poisson equation for stream function (8) is solved using the conjugate gradient scheme without preconditioning (CG). The potential values are used to calculate the new velocities $\left(u^{n+1}, v^{n+1}\right)$ via the discrete form of equation (9).

For the primitive variables approach, all the variables are solved on a single (2D or 3D) finite volume mesh. The mesh need not be uniform. All the variables are stored at the grid points $-\mathrm{a}$ collocated grid. The scheme for solving for the velocity field is based on the work of Ferziger and Perić, ${ }^{25}$ where complete details may be found. The velocity field on the collocated grid is solved using the SIMPLE algorithm. Staggered grids are traditionally used with this algorithm in order to avoid instability with pressure correction. However, it can be shown that judicious interpolation of the pressure data will result in a collocated grid algorithm that is not susceptible to pressure oscillations. This is the scheme used in the present work. In recent years, there has been a move towards using collocated variables that are more adaptable to solving problems in 
irregular geometries with unstructured grids. It is hoped that such features will be incorporated in extensions to this work. Scheme II can be summarized as follows:

- The mass conservation (Eq. 1), momentum (Eq. 2) and energy equations (Eq. 3) are solved using a sequential solution algorithm with under-relaxation and outer iterations to couple the two in the general case for an alloy model. In accordance with the SIMPLE algorithm, deferred correction is used for the mass fluxes (a so-called Picard iteration ${ }^{25}$ ) due to the nonlinear nature of the momentum equations and correction by the pressure correction equation. Deferred correction is also used in the source terms for both equations. SOR or (more often) MSI iteration is used for the inner iterations. CDS interpolation is used for all convective fluxes, also with deferred correction to preserve diagonal dominance of the coefficient matrix. Once convergence is achieved, all the solution vectors at $n+1$, i.e., velocity $\left(\tilde{U}^{n+1}\right)$, temperature $\left(T^{n+1}\right)$ and liquid fraction $\left(f_{l}^{n+1}\right)$, are known. The same small freezing range as employed in scheme I is also needed here.

- No front reconstruction is needed. Velocities are extinguished in the solid due to the BlakeCozeny-Karman ${ }^{22}$ source term in the momentum equations. This source term is capped at a maximum value of $1 \times 10^{8}$ in the solid region in order to prevent an overflow error.

Both schemes can also incorporate the physics involved with alloy solidification (such as solving a concentration equation with an appropriate physical model for handling the redistribution of solute at the solid/liquid interface ${ }^{28}$ ); however this feature is not required for the present study where the solidification of a pure material is being considered. 


\section{NUMERICAL RESULTS AND COMPARISON WITH EXPERIMENT}

\section{Two-Dimensional Simulations}

For the no-growth case (fronts shown in Fig. 7 and velocities in Fig. 9), flow visualization showed that the convective flow, while containing measurable $3 \mathrm{D}$ effects, is primarily $2 \mathrm{D}$ in nature. It is therefore expected that a 2D Cartesian numerical simulation, with the computational domain selected as the mid-vertical plane, is an adequate model of the process. Results for such 2D simulations are provided here. In the next section, results for a fully 3D Cartesian simulation of the process will be presented, and the utility of the 2D model discussed.

Results were obtained using both Schemes I and II. The computational domain and boundary conditions are shown in Fig. 10. For the boundary conditions, the no-growth temperature profiles from de Groh and Lindstrom ${ }^{2}$ were used. The simulation domain extends from $\mathrm{z}=-19$ $\mathrm{mm}$ in the solid to $+40 \mathrm{~mm}$ in the melt. Since there are only small temperature gradients further into the melt, numerical experiments have shown that convective velocities are not substantially changed by lengthening the domain. The material properties used for SCN and the borosilicate glass ampoule are given in Table 1. A grid-independence study for both the $\zeta$ - $\psi$ function and $P$ $U$ formulations was performed, leading to the selection of the preferred mesh of $200 \times 28$ cells for both techniques.

Figure 11 is a plot of the velocity vectors and isotherms for the pure SCN case found using (a) the $\zeta-\psi$ function scheme and (b) the $P-U$ scheme for convective velocities. The dashed line is the interface location. A single, clockwise rotating longitudinal convective cell has formed in the melt. Warm bulk fluid moves along the top wall and washes on to the top of the interface. The fluid then falls toward the bottom wall and is convected away. The interface takes on a distinctly curved shape with the solid being concave. This shape is due to the influence of convection on the interface shape; warm fluid introduced to the interface near the top wall acts to melt the 
interface back. As the fluid cools and falls toward the bottom wall the interface is distorted less. For the case shown in Fig. 11(a), the maximum convective velocity was found to be $1.515 \mathrm{~mm} / \mathrm{s}$ at the location $(-1.30,+0.90)$, which is near the interface where applied temperature gradients are steepest. This value agrees well with the experimentally measured value of $1.50 \pm 0.08 \mathrm{~mm} / \mathrm{s}$ at the same location. The influence of the ampoule walls on the process may also be noted; the difference between the temperatures on the inside and outside of the walls is largest near the interface. At $z=0$ the temperature differences are $2.14 \mathrm{~K}$ and $1.23 \mathrm{~K}$ for the top and bottom walls, respectively. As can be seen in Fig. 11, the results found from both simulations are in good agreement overall. However the $P-U$ results tend to have higher convective velocities. The differences between both sets of results are better appreciated by examining the front locations.

The experimentally and numerically determined interface locations are compared in Fig. 12. Experimental data for the MVP, as shown in Fig. 7, are also reproduced here. Considering the comparison between the numerically calculated and measured interface shapes, the results agree to within a maximum discrepancy of $0.47 \mathrm{~mm}$. This is acceptable agreement given the variability of the measured temperature boundary conditions $\left( \pm 1^{\circ} \mathrm{C}\right)$ and the experimental error in determining the interface $( \pm 0.2 \mathrm{~mm})$. When considering the two numerically predicted interface shapes, the agreement between the two methods is reasonable. The $\zeta-\psi$ algorithm exhibits slightly better overall agreement, with over half of the results exhibiting a maximum discrepancy of $0.25 \mathrm{~mm}$. In the region of approximately $-0.8<y<2.4 \mathrm{~mm}$, the difference between the two sets of results is largest. A possible reason for this difference is the different ways the two methods handle the solid/liquid front. For the $\zeta-\psi$ solution scheme, boundary conditions for the velocity field are explicitly applied on a reconstructed front, whereas the $P-U$ scheme relies on a momentum source term. The differences in convective velocities calculated 
by the two algorithms (maximum values of $1.627 \mathrm{~mm} / \mathrm{s}$ for $P$ - $U$ versus $1.515 \mathrm{~mm} / \mathrm{s}$ for $\zeta-\psi$ ) also play a role in the differences in predicted front shape (and vice versa).

\section{Three-Dimensional Simulations}

Full three-dimensional Cartesian simulations were performed next. Since the $\zeta-\psi$ solution scheme does not extend to the $3 \mathrm{D}$ case, all the results presented in this subsection use the $P-U$ scheme for solving the velocity field. Heat conduction through the ampoule walls is included in the calculations.

Figure 13(a) shows a schematic of the computational domain. Identical domains were used for both no-growth and $40 \mu \mathrm{m} / \mathrm{s}$ growth cases. The problem is symmetric about the $x=0$ plane; as a result, calculations were only performed in half the domain. Experimentally measured temperature traces were used as the thermal boundary conditions on the outside of the ampoule. Around the periphery of the ampoule temperature values are found by linearly interpolating between the experimentally measured values, except for on the bottom of the ampoule, which is assumed to have a temperature profile equal to the bottom middle thermocouple reading, as illustrated in Fig. 13(b). For the no-growth case, temperature data from de Groh and Lindstrom ${ }^{2}$ were used, while for the $40 \mu \mathrm{m} / \mathrm{s}$ growth case, the temperature data shown in Fig. 6 were used. As for the $2 \mathrm{D}$ case, grid independence was established for the 3D simulations. For the nogrowth case, $14 \times 36 \times 180$ cells were employed, with $14 \times 36 \times 200$ cells being used for the 40 $\mu \mathrm{m} / \mathrm{s}$ growth case. Both meshes were graded in order to better resolve the velocities in the near wall region and refined near the interface. For the $40 \mu \mathrm{m} / \mathrm{s}$ growth case there was a larger section of finer cells since the interface moves with time; the mesh had to be fine enough to resolve the interface over a larger length.

Velocity vectors and isotherms for this case are shown in Fig. 14. Figure 14(a), (b), (c) and (d) are plots through the (vertical) $x=0,-1.5$ and $-2.8 \mathrm{~mm}$ and (horizontal) $y=+1.2 \mathrm{~mm}$ planes, 
respectively. In these plots, velocity vectors are shown at every third mesh point in the $z$ direction for clarity. At first glance, the center plane $(x=0$, Figure 14a), results seem similar to the 2D case (Fig. 11). However there are some noticeable differences. For the 3D case, the isotherms are not as sharply distorted out into the bulk flow. This indicates that the 2D model predicts higher convective velocities overall. This is as expected; the front and back walls exert a shear force on the flow which cannot be calculated by the $2 \mathrm{D}$ model. The results in the $x=$ $-1.5 \mathrm{~mm}$ plane (Figure 14b) are similar to those for the center plane. The only discernible difference is that the path traveled by the flow near the interface is more vertical since the interface is deflected less. Near the wall at $x=-2.8 \mathrm{~mm}$ (Figure 14c), the flow is in the viscous layer and convective velocities are significantly lower, as is the interface distortion.

Predicted values for velocities were obtained at the same locations as for the measurements shown in Fig. 9. Since the measurement points do not coincide with the finite-volume centers used for the simulation, interpolation was used to find the velocity values. Agreement between the numerical and experimental velocity values was good: the predicted velocities were all within $0.17 \mathrm{~mm} / \mathrm{s}$ of the experimental measurements, with over $60 \%$ of the values agreeing to within $0.08 \mathrm{~mm} / \mathrm{s}$. Also the maximum measured velocity in the ampoule $(1.50 \mathrm{~mm} / \mathrm{s})$ compares well with the maximum simulated velocity of $1.47 \mathrm{~mm} / \mathrm{s}$.

A comparison between experimentally determined and numerically calculated interface locations is presented in Fig. 15. The front shapes in the three $x-z$ (horizontal) planes shown in Fig. 15(a) (acronyms MHP, BHP and THP are as explained in connection with Figs. 4 and 7) show that the experimental and numerical interface locations agree to within $0.25 \mathrm{~mm}$. The general features of the front shapes in the two $y-z$ (vertical) planes shown in Fig. 15(b) are as follows. The interface is considerably distorted from the vertical, with the solid forming a concave shape. Again, the predicted front locations agree well with the experimental results, 
with the maximum deviation being approximately $0.4 \mathrm{~mm}$; this is within the total experimental error for the interface location determination due to uncertainties in thermocouple location and interface measurement. Overall, the agreement both in terms of interface shape and melt velocities is better than for the $2 \mathrm{D}$ model of the same process.

Bridgman growth at $40 \mu \mathrm{m} / \mathrm{s}$ was simulated next. Here, the thermal jackets translate at a steady velocity rendering the solution time-dependent. To simulate the movement of these jackets, the thermal boundary condition was translated at the same steady velocity. A steadystate solution (with the boundary temperatures immobile) was used as the initial condition. As the simulation proceeds, the numerically determined interface begins to elongate as the temperatures in the center "lag" behind those applied on the outside of the ampoule wall. After $t$ $\approx 200 \mathrm{~s}$, the interface shape becomes steady and the interface as a whole is pulled along by the boundary temperatures moving at $40 \mu \mathrm{m} / \mathrm{s}$.

Velocity vectors and isotherms for this case are shown in Fig. 16. Slices through vertical planes are shown in Fig. 16(a)-(c), while a slice through a horizontal plane is shown in Fig. 16(d). Qualitatively, the convective patterns are similar to the no-growth case with some key differences. Firstly, the flow is much more three-dimensional. The flow patterns shown in Fig. 16(a) (slice through the symmetry plane) are noticeably different from those shown in Fig. 16(b) (slice mid-way between the symmetry plane and wall). For the no-growth case, the results through these two planes (Figs. 14a and b) were almost identical. This is a result of the more distorted interface shape and isotherms caused by translation of the thermal boundary conditions. The velocity vectors shown in the horizontal slice (Fig. 16d) are also much more interesting than for the no-growth case. As the flow approaches the interface, there are noticeable velocity components in the $x$ direction. These components indicate that the flow is pushed out away from 
the centerline as the flow approaches the interface. The flow patterns and strengths indicated by the velocity vectors are in good agreement with the flow visualizations.

Interface shapes for this $40 \mu \mathrm{m} / \mathrm{s}$ growth case are shown in Fig. 17. Figure 17(a) shows the interface shape in the mid horizontal plane (MHP, $y=+1.2 \mathrm{~mm}$ ). It may be recalled that interface locations in the other horizontal planes could not be determined quantitatively from the experiments. Figure 17(b) contains interface shapes in the MVP. The experimental data are

from the present study (Fig. 8b) as well as from de Groh and Lindstrom ${ }^{2}$ to give an indication of the error that may be expected from the experiment. The numerical predictions, while exhibiting good agreement with the experiments, tend to predict a more dramatic curvature than that found experimentally. The strongly distorted shape of the interface indicates the 3D nature of the heat and fluid flow in this growth problem; using a 2D model clearly would not yield a faithful simulation of the process despite providing reasonable results (although inferior to the 3D model) for the no-growth case. The distortion of the interface in the horizontal $(x-z)$ plane for the no-growth case was much less pronounced such that a 2D approach was more reasonable for that problem than in the growth case.

\section{CONCLUSIONS}

A combined experimental and computational study of the directional solidification of pure SCN under both no-growth and growth conditions has been performed. This combined experimental-numerical database has revealed the role of convection on influencing interface shape (and hence the final properties of the solidified material) during Bridgman growth.

The experiments were carefully designed with the aim of creating a complete database for the validation of numerical algorithms that simulate solidification. The boundary temperature profiles, interface shapes and convective velocities were measured. The experimental results 
indicated the following. The interface shapes for pure SCN are stable and non-dendritic; however they are not flat but instead assume a complex three-dimensional shape. This indicates the presence of thermally driven convective motion in the melt region. Flow visualization for the pure SCN sample indicates that a single longitudinal convective cell forms, with warm fluid approaching the interface from the top of the domain, falling at the cool interface and retreating along the bottom of the ampoule. This cell is primarily two-dimensional - the only secondary flows observed occur near the interface and act to push approaching fluid out from the centerline toward the side walls. Convective velocities under no-growth conditions were measured using seed particles tracked by two orthogonal video cameras. The maximum velocity was measured to be $1.50 \pm 0.08 \mathrm{~mm} / \mathrm{s}$. These detailed velocity measurements, coupled with the flow visualization, are a key contribution of the present study. While it has been recognized in the literature that convective flow plays a critical role in the solidification process, there are few instances of accurate and detailed experimental measurements of such velocities. In the present study, the flow field has been carefully quantified so that the role of velocity can be fully understood.

A series of both 2D and 3D numerical simulations of the process further elucidate the key role of convection on the interface shape and hence the solidification process. For the no-growth case, the convective cell is primarily two-dimensional in nature, and so a 2D model is a good representation of the process. Two-dimensional simulations using two different algorithms were performed. The results for both algorithms agreed well with the experimental data, with the vorticity-stream function algorithm providing slightly better agreement than the pressurevelocity formulation. A three-dimensional simulation of the no-growth case gave similar good agreement with experiment: over $60 \%$ of the predicted velocities agreed with the experimental velocity measurements to within experimental error. 
For the growth case, the convective cell (and interface shape) is much more strongly threedimensional than for the no-growth case, and so a $2 \mathrm{D}$ model is not appropriate. Threedimensional numerical simulations of this process agree reasonably with experimental results in terms of predicted interface shapes. The simulations reveal the nature of the convective flow which was not able to be experimentally measured for the growth case.

Both numerical formulations used in this study (vorticity-stream function and pressurevelocity) are able to faithfully simulate phase-change problems. While the vorticity-stream function method gives superior results for $2 \mathrm{D}$ problems, it cannot readily be extended to three dimensions, severely limiting its utility. In ongoing work, the pressure-velocity based scheme is being used to simulate transient 3D melting and solidification in metals and metallic alloys. 


\section{REFERENCES}

1) Flemings, M. C., Solidification Processing, McGraw-Hill, 1974.

2) de Groh III, H. C. and Lindstrom, T., "Interface shape and convection during solidification and melting of succinonitrile," NASA Tech Memo. 106487, June 1994.

3) Garimella, S. V., McNulty, J. P. and Schlitz, L. Z., "Formation and suppression of channels during upward solidification of a binary mixture," Metall. and Mater. Trans. A, Vol. 26A, pp. 971-981, 1995.

4) Magirl, C. S. and Incropera, F. P., "Flow and morphological conditions associated with unidirectional solidification of aqueous ammonium chloride," J. Heat Transfer, Vol. 115, pp. 1036-1043, 1993.

5) Hellawell, A., Sarazin, J. R. and Stuebe, R. S., "Channel convection in partly solidified systems," Phil. Trans. R. Soc. London, Vol. 345A, pp. 507-544, 1993.

6) Copley, S. M., Giamei, A. F., Johnson, S. M. and Hornbecker, M. F., "The origin of freckles in unidirectionally solidified castings,” Met. Trans., Vol. 1, pp. 2193-2204, 1970.

7) de Cheveigné, S., Guthmann, C., and Lebrun, M.-M., "Nature of the transition of the solidification front of a binary mixture from a planar to a cellular morphology," J. Crystal Growth, Vol. 73, pp. 242-244, 1985.

8) Eshelman, M. A., Seetharaman, V. and Trivedi, R., "Cellular spacings-I. steady-state growth," Acta Met., Vol. 36, pp. 1165-1174, 1988.

9) Eshelman, M. A., Seetharaman, V. and Trivedi, R., "Cellular spacings-II. dynamical studies," Acta Met., Vol. 36, pp. 1175-1185, 1988.

10) Adornato, P. M. and Brown, R. A., "Convection and segregation in directional solidification of dilute and non-dilute binary alloys," J. Crystal Growth, Vol. 80, pp. 155190, 1987.

11) Liang, M. C. and Lan, C. W., "Three-dimensional convection and solute segregation in vertical Bridgman crystal growth," J. Crystal Growth, Vol. 167, pp. 320-332, 1996.

12) Simpson, J. E. and Garimella, S. V., "An investigation of the solutal, thermal and flow fields in unidirectional alloy solidification," Int. J. Heat Mass Transfer, Vol. 41, pp. 2485$2502,1998$.

13) Zeng, $X$. and Faghri, A., "Temperature-transforming model for binary solid-liquid phasechange problems part II: Numerical simulation," Numer. Heat Transfer, Vol. 25B, pp. 481500, 1994. 
14) Yao, M. and de Groh III, H. C., "Three-dimensional finite element method simulation of Bridgman crystal growth and comparison with experiments," Numer. Heat Transfer, Vol. 24A, pp. 393-412, 1993.

15) Yeoh, G. H., de Vahl Davis, G., Leonardi, E., de Groh III, H. C. and Yao, M., "A numerical and experimental study of natural convection and interface shape in crystal growth," $J$. Crystal Growth, Vol. 173, pp. 492-502, 1997.

16) de Groh III, H. C. and Yao, M., "Numerical and experimental study of transport phenomena in directional solidification of succinonitrile," Transport Phenomena in Solidification, ASME HTD-Vol. 284, pp. 227-243, 1994.

17) Chopra, M. A., Glicksman, M. E. and Singh, N. B., "Dendritic solidification in binary alloys," Met. Trans., Vol. 19A, pp. 3087-3096, 1988.

18) Simpson, J. E., de Groh III, H. C. and Garimella, S. V., "Directional solidification of pure succinonitrile and a succinonitrile-acetone alloy," NASA Tech. Mem. 2000-209381/REV1, September 2000 .

19) Mennetrier C., Chopra, M. A. and de Groh III, H. C., "Effect of thermal convection on the shape of a solid-liquid interface," Forum on Microgravity Flows, ASME FED-Vol. 111, pp. 5-10, 1991.

20) Merchant, G. J. and Davis, S. H., "Shallow cells in directional solidification," Physical Review Letters, Vol. 63, pp. 573-575, 1989.

21) Dantzig, J. A., "Modeling liquid-solid phase changes with melt convection," Int. J. Numer. Meth. in Engineering, Vol. 28, pp. 1769-1785, 1989.

22) Oldenburg, C. M. and Spera, F. J., "Numerical modeling of solidification and convection in a viscous pure binary eutectic system," Int. J. Heat Mass Transfer, Vol. 34, pp. 2107-2121, 1991.

23) Hirasaki, G. J and Helums, J. D., "A general formulation of the boundary conditions on the vector potential in three-dimensional hydrodynamics," Quart. Appl. Math., Vol. 16, pp. 331-342, 1968.

24) Roache, P. J., Computational Fluid Dynamics, Hermosa Press, Albuquerque, 1976.

25) Ferziger, J. A., and Perić, S. M., Computational Methods for Fluid Dynamics, Springer Verlag, 1995.

26) Hirt, C. W. and Nichols, B. D., "Volume of fluid (VOF) method for the dynamics of free boundaries," J. Comp. Phys., Vol. 39, pp. 210-255, 1981.

27) Samarskii, A. A. and Andreyev, V. B., "On a high-accuracy difference scheme for an elliptic equation with several space variables," USSR Comput. Math. and Math. Phys., 1963, Vol. 3, pp.1373-1382. 
28) Simpson, J. E. and Garimella, S. V., "The influence of gravity levels on the horizontal Bridgman crystal growth of an alloy," Int. J. Heat Mass Transfer, Vol. 43, pp. 1905-1923, 2000.

29) Paradies, C. J., The Influence of Forced Convection during Solidification on Fragmentation of the Mushy Zone of a Casting, Ph.D. Dissertation, Rensselaer Polytechnic Institute, 1993. 


\section{FIGURE CAPTIONS}

Fig. 1. Schematic of NASA's Transparent Directional Solidification Furnace (TDSF). An additional CCD camera setup (including camera, digital video tape recorder, digital video disc recorder and monitor like the one pictured) is not shown for clarity. This camera points directly down into the page. The position of the camera is indicated by the broken outline.

Fig. 2. Photographs of (a) overview of the experimental setup, including the CCD cameras in the vertical and horizontal planes, and (b) close-up of the copper heating and cooling jackets (with insulation removed to reveal the tubing). The gap between the heating and cooling jackets serves as the gradient zone. Both the hot and cold jackets can temporarily slide left or right to permit viewing of the interface.

Fig. 3. Schematics of ampoule instrumentation: (a) cross-section of ampoule showing thermocouple locations and definition for $x-y$ coordinates, (b) pure SCN ampoule instrumented with five thermocouples, and (c) schematic of ampoule mounted in furnace; $z=0$ is taken to be in the center of the gap between the hot and cold jackets.

Fig. 4. Sample images used to measure interface positions: (a) $x-z$ and (b) $y-z$ planes (THP, MHP, BHP are top, mid and bottom horizontal planes, FVP, MVP are front and midvertical planes).

Fig. 5. Samples of the images used to estimate the flow velocities for the no-growth case, (a) top view ( $x-z$ planes) and (b) side view ( $y-z$ planes). For reproducibility, the images have been inverted so that dark becomes light and vice versa. The larger dots and streaks are reflections from defects in the glass, while the smaller dots are the seed particles. The interface locations in the mid planes can be clearly seen, as can the edges of the hot and cold jackets.

Fig. 6. Temperature profiles at a growth rate of $40 \mu \mathrm{m} / \mathrm{s}$. Thermocouple locations are indicated in Fig. 3. The $z=0$ position corresponds to the center of the $5 \mathrm{~mm}$ gradient zone.

Fig. 7. Front locations under no-growth conditions in (a) horizontal and (b) vertical planes. The interface is stable and non-dendritic but noticeably distorted from the vertical. The solid is concave with a symmetric crescent-like section when viewed from above and asymmetric and thicker towards the bottom when viewed from the side.

Fig. 8. Front locations at a growth rate of $40 \mu \mathrm{m} / \mathrm{s}$ in (a) horizontal and (b) vertical planes.

Fig. 9. Measured velocities during no-growth in (a) horizontal and (b) vertical planes.

Fig. 10. Computational domain and applied thermal boundary conditions along the exterior of the top and bottom ampoule walls. These boundary conditions are from thermocouple readings for the TM and BM thermocouples (refer to Fig. 3).

Fig. 11. Isotherms, velocity vectors and front locations for 2D simulations of solidification under no-growth conditions: (a) vorticity-stream function and (b) pressure-velocity results. 
Fig. 12. Comparison of measured and predicted (from 2D simulations) interface shapes under no-growth conditions.

Fig. 13. (a) Problem domain for the 3D simulations, (b) thermal boundary conditions around the periphery of the ampoule obtained by linearly interpolating measured temperatures (from de Groh and Lindstrom ${ }^{2}$ for no-growth, and from the data in Fig. 6 for $40 \mu \mathrm{m} / \mathrm{s}$ growth).

Fig. 14. Velocity vectors and isotherms for 3D simulations under no-growth conditions: (a) vertical slice through symmetry plane $(x=0)$, (b) vertical slice halfway between center plane and rear wall $(x=-1.5 \mathrm{~mm}),(\mathrm{c})$ vertical slice through a plane near the rear wall $(x$ $=-2.8 \mathrm{~mm}$ ), and (d) horizontal slice through $y=+1.2$. Vectors are shown at every third mesh point in the $z$ direction for clarity.

Fig. 15. Comparison of predicted (from 3D simulations) and measured interface shapes (as in Fig. 7) under no-growth conditions.

Fig. 16. Velocity vectors and isotherms for 3D simulations under $40 \mu \mathrm{m} / \mathrm{s}$ growth conditions after $t=300 \mathrm{~s}$ : (a)-(d) refer to the same vertical and horizontal slices as in Fig. 14.

Fig. 17. Comparison of interface shapes for the $40 \mu \mathrm{m} / \mathrm{s}$ growth case. 
Table 1: Thermophysical properties for $\mathrm{SCN}^{29}$ and the borosilicate glass ampoule ${ }^{16}$

\begin{tabular}{|lll|}
\hline & Property & Value \\
\hline \hline SCN liquid & $k_{l}$ & $0.223 \mathrm{~W} / \mathrm{mK}$ \\
& $c_{p l}$ & $2000 \mathrm{~J} / \mathrm{kgK}$ \\
& $\rho$ & $990 \mathrm{~kg} / \mathrm{m}^{3}$ \\
& $\Delta H$ & $46.24 \mathrm{~J} / \mathrm{kg}$ \\
& $\mu$ & $3.0 \times 10^{\square 3} \mathrm{Ns} / \mathrm{m}$ \\
& $\beta_{T}$ & $8.1 \times 10^{\square 4} 1 / \mathrm{K}$ \\
\hline SCN solid & $k_{s}$ & $0.225 \mathrm{~W} / \mathrm{mK}$ \\
& $c_{p s}$ & $1955 \mathrm{~J} / \mathrm{kgK}$ \\
& $\rho$ & $990 \mathrm{~kg} / \mathrm{m}^{3}$ \\
& $T_{m}$ & $58.08{ }^{\circ} \mathrm{C}$ \\
\hline Borosilicate glass & $k_{w}$ & $1.2 \mathrm{~W} / \mathrm{mK}$ \\
& $c_{p w}$ & $753.5 \mathrm{~J} / \mathrm{kgK}$ \\
& $\rho_{w}$ & $2300 \mathrm{~kg} / \mathrm{m}^{3}$ \\
\hline
\end{tabular}




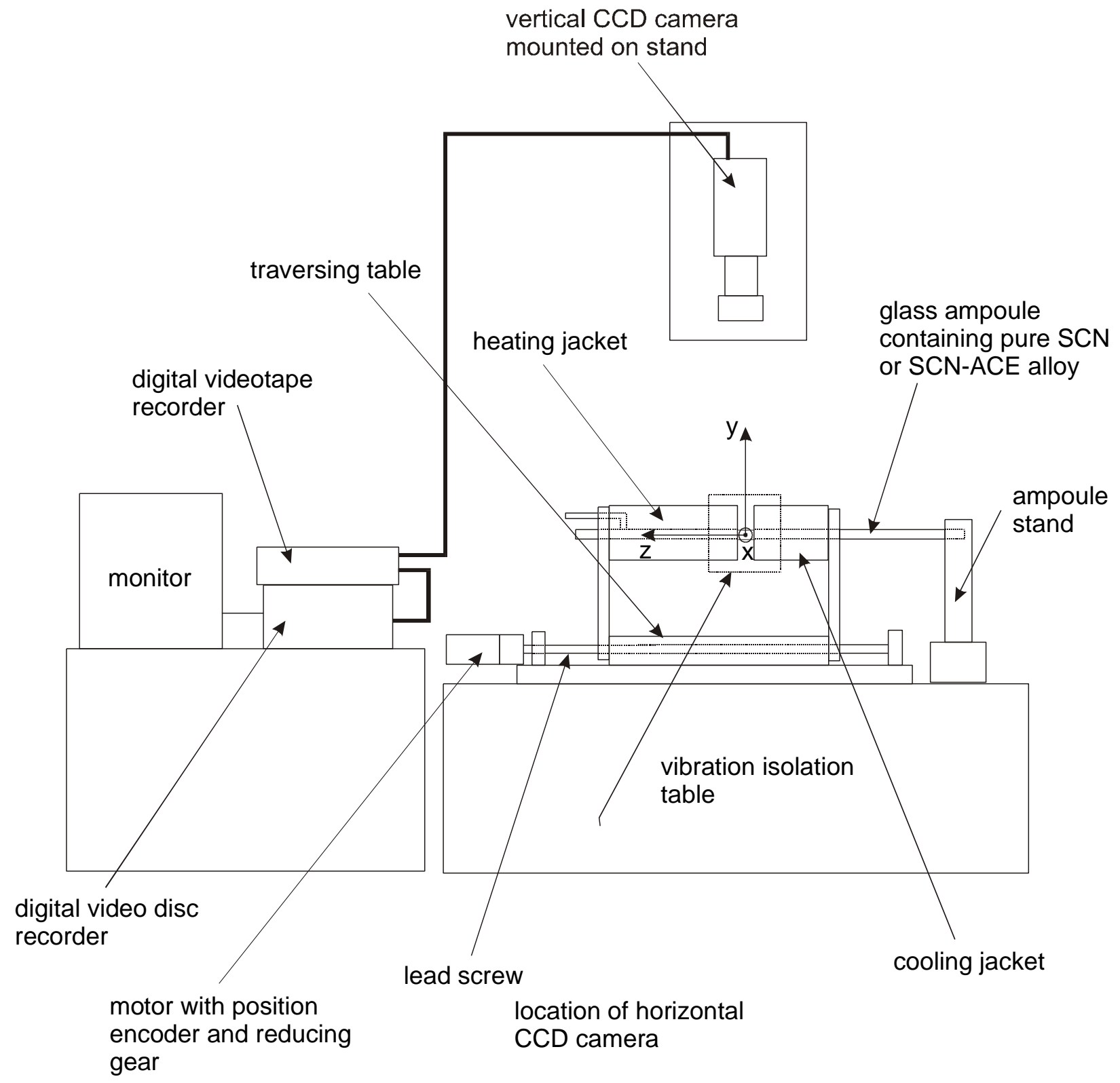

Fig. 1, Simpson et al. 

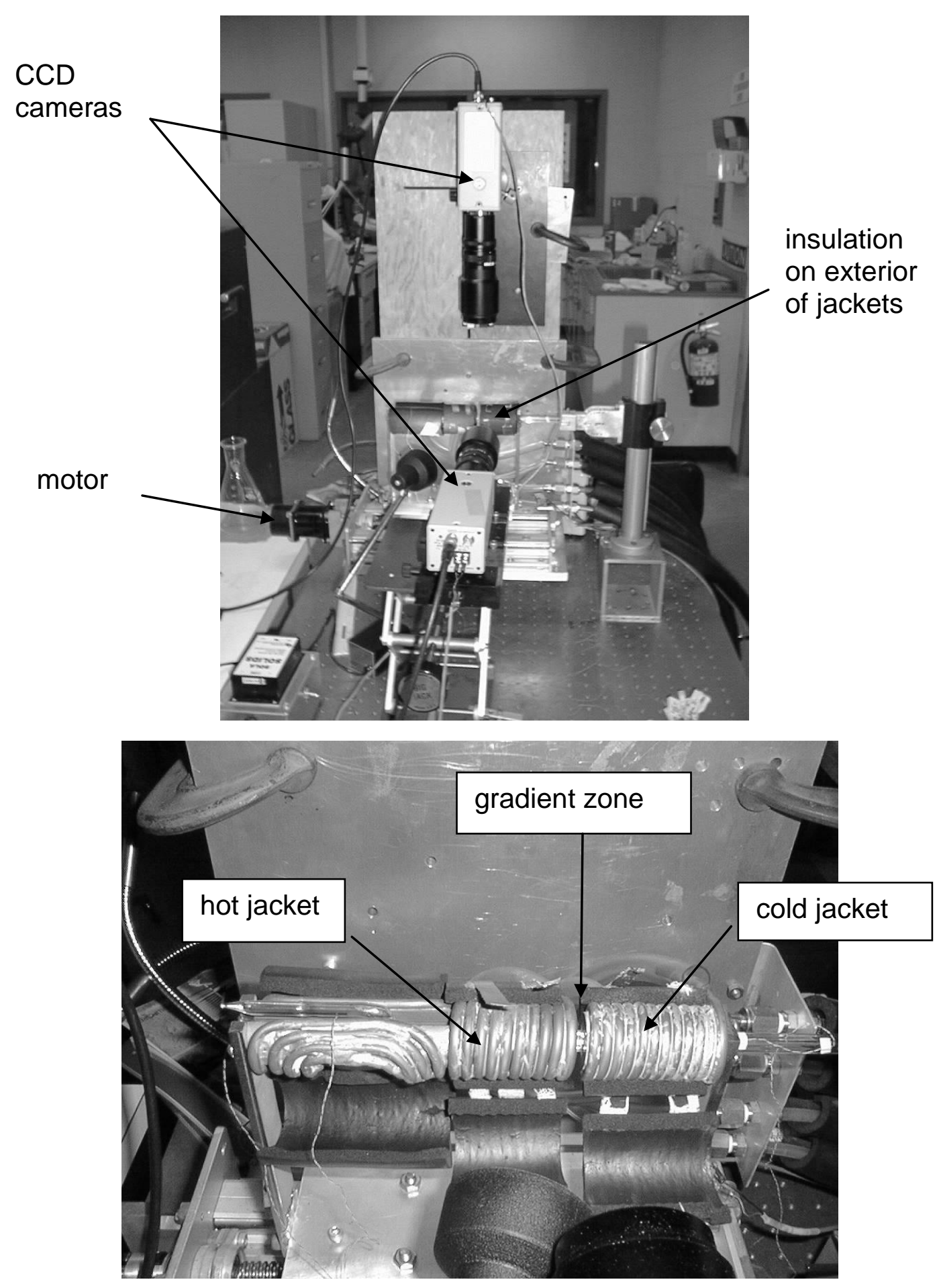

Fig. 2, Simpson et al. 


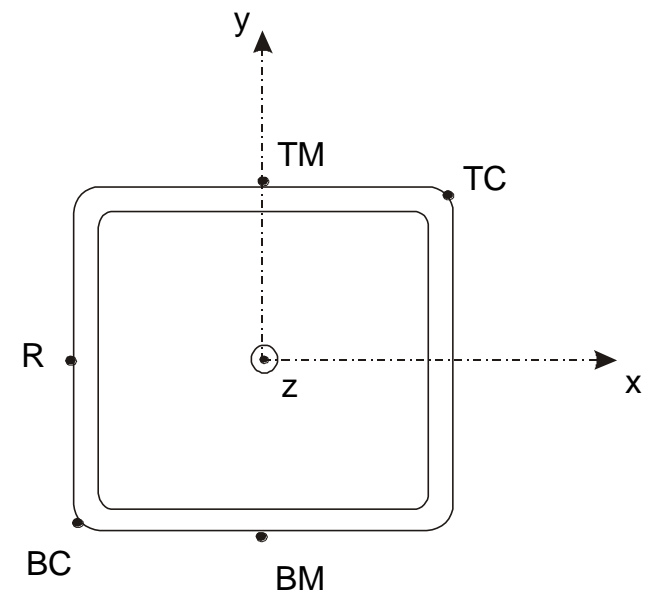

(a)

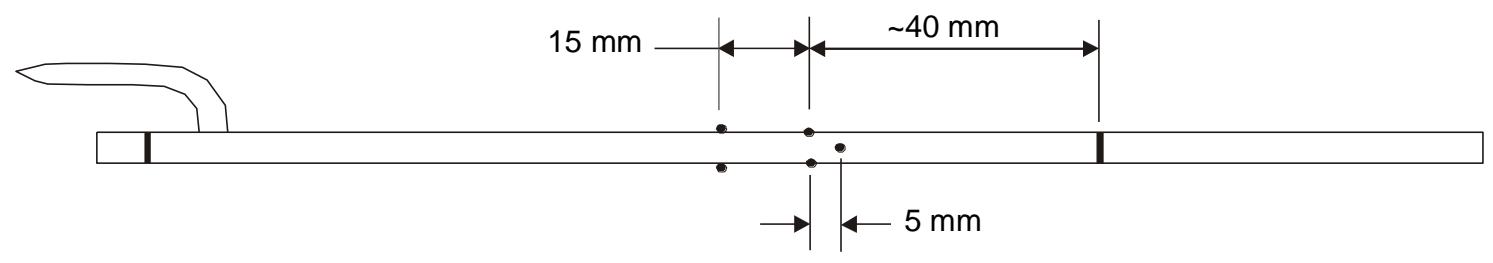

(b)

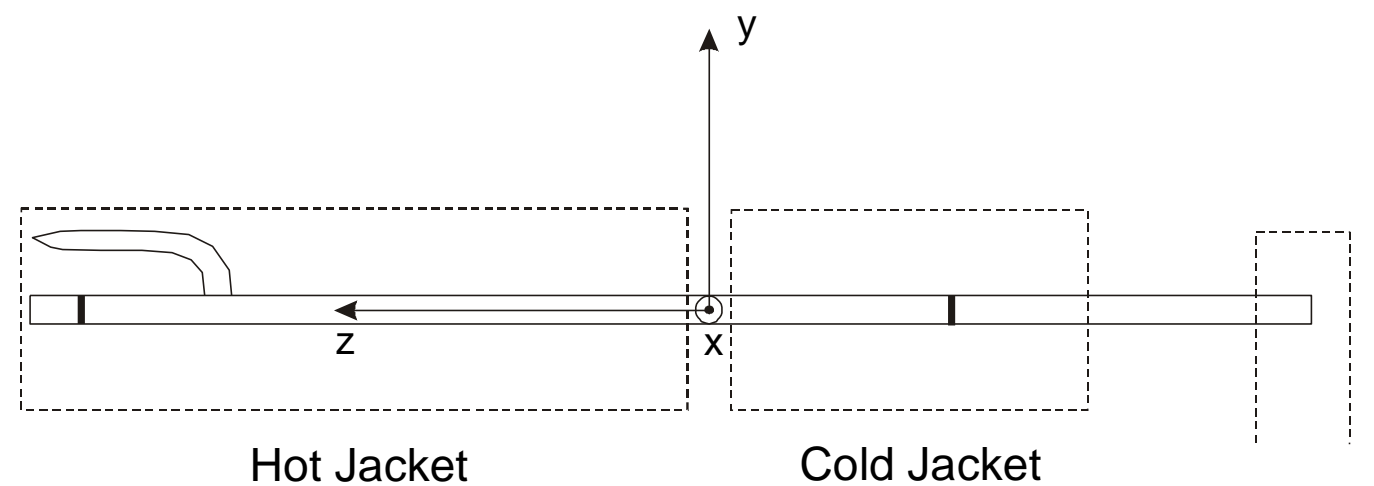

(c)

Fig. 3, Simpson et al. 
TOP VIEW
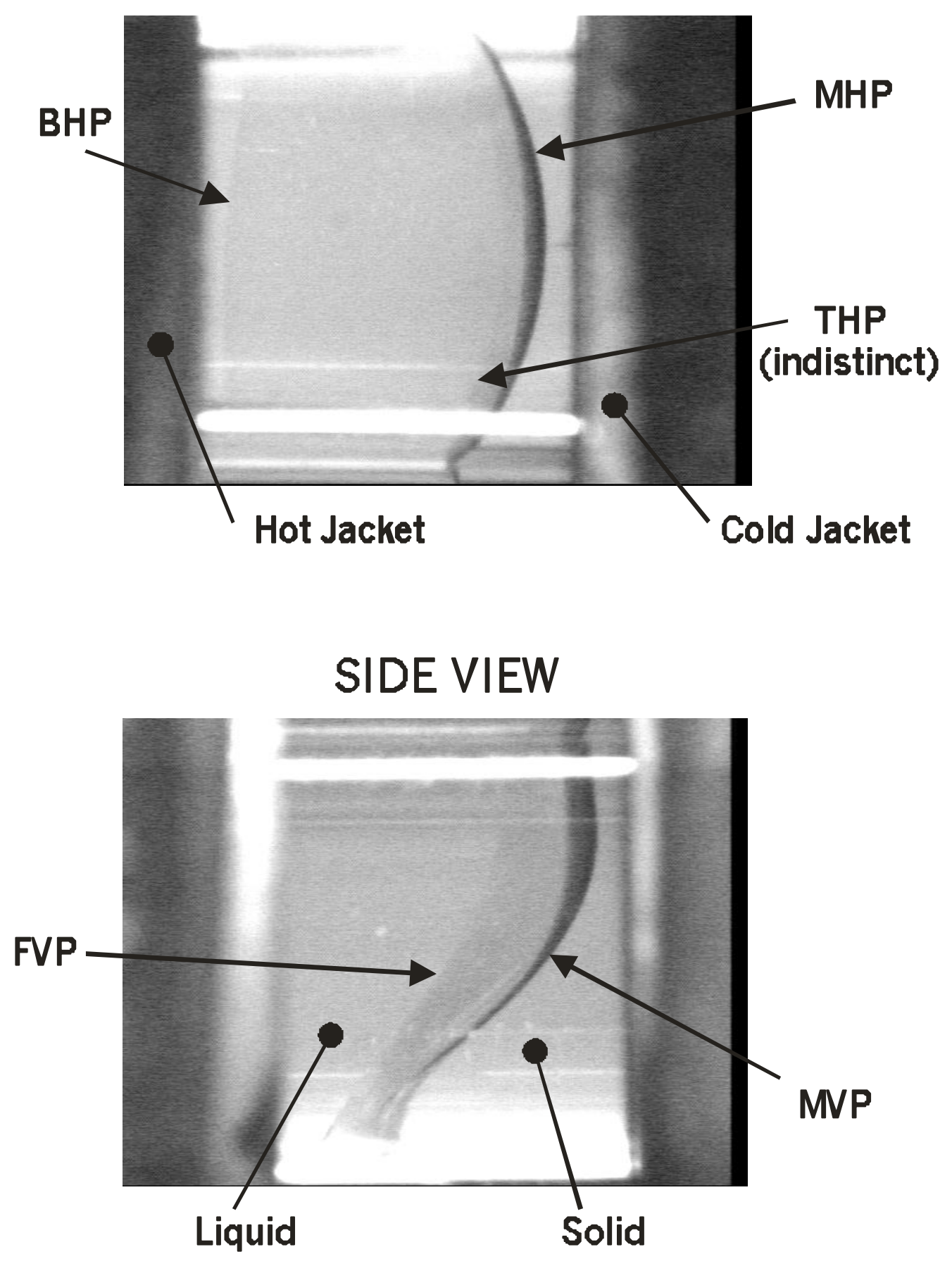

Fig. 4, Simpson et al. 


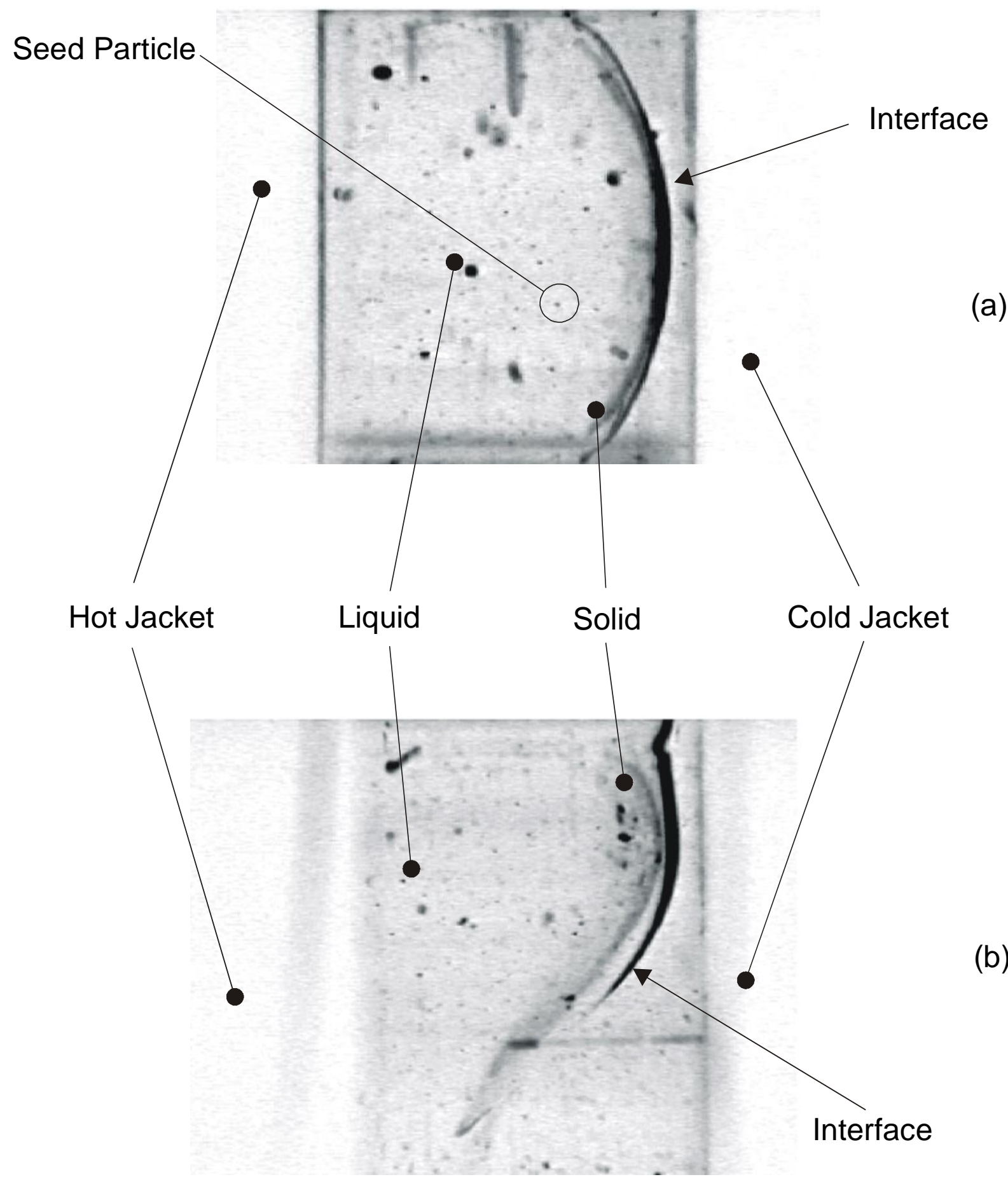

Fig. 5, Simpson et al. 


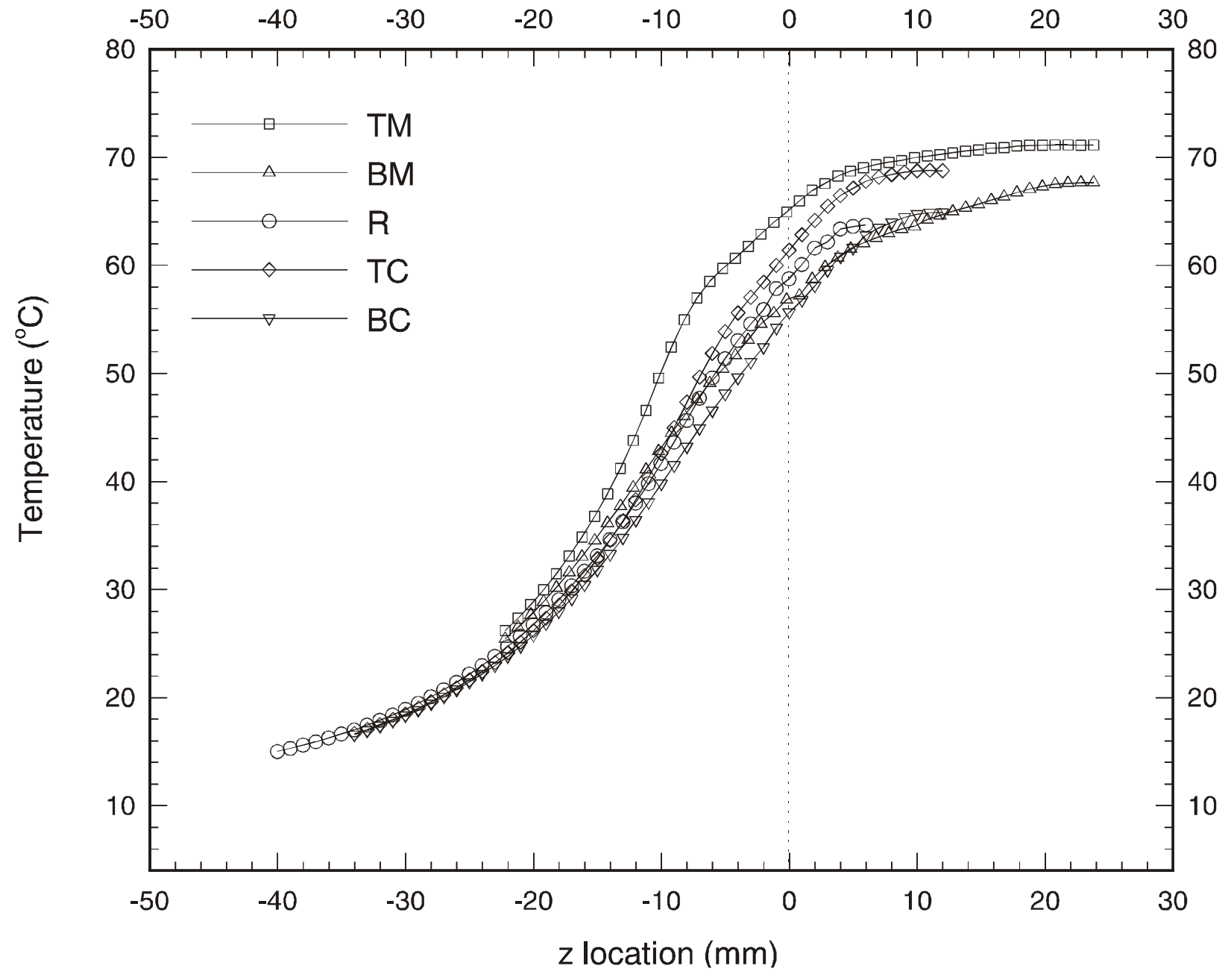

Fig. 6, Simpson et al. 


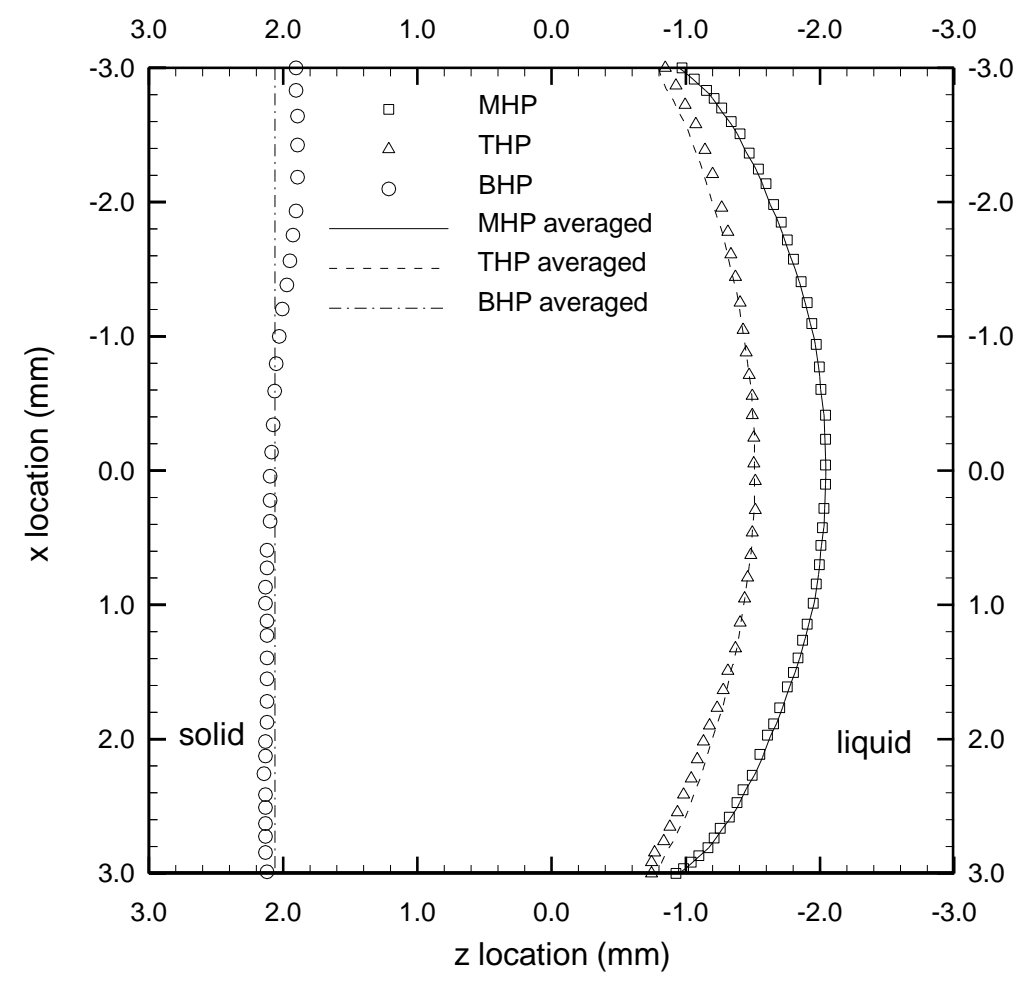

(a)

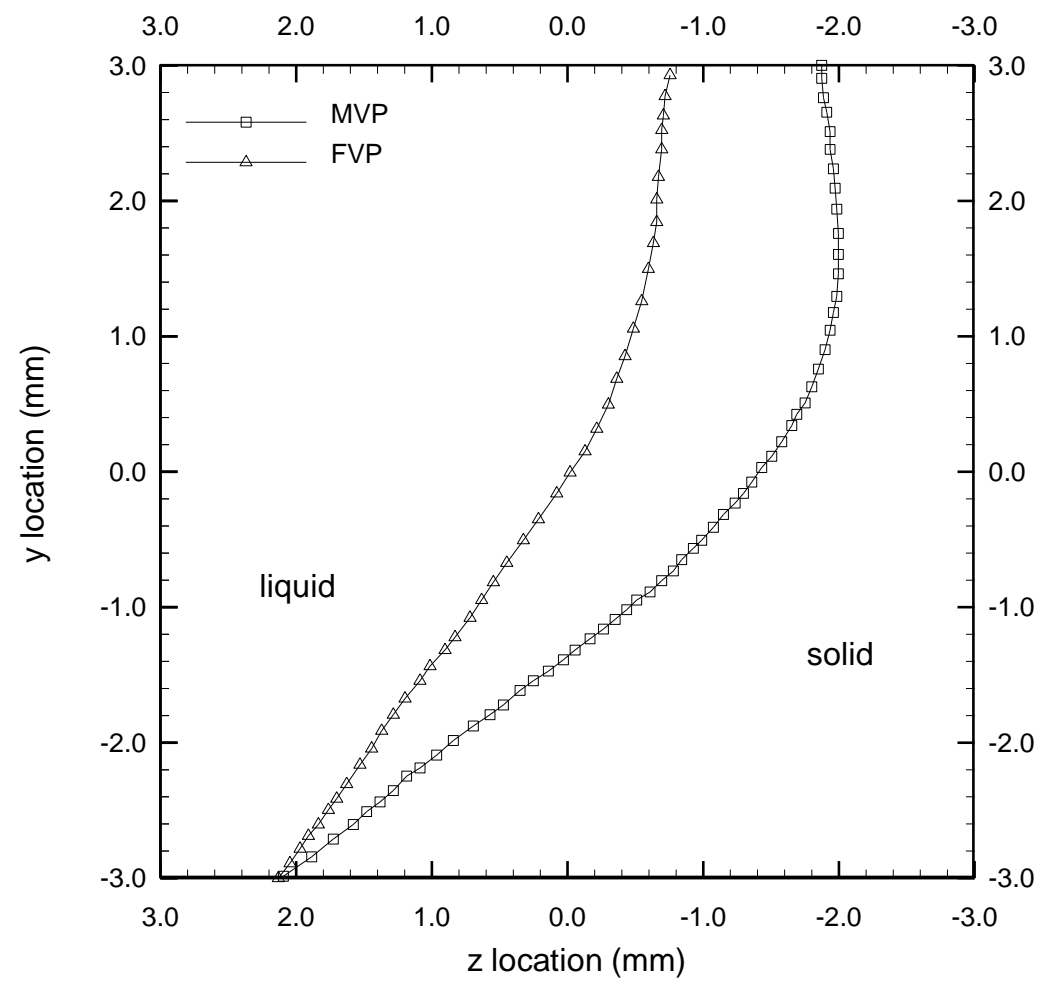

(b)

Fig. 7, Simpson et al. 


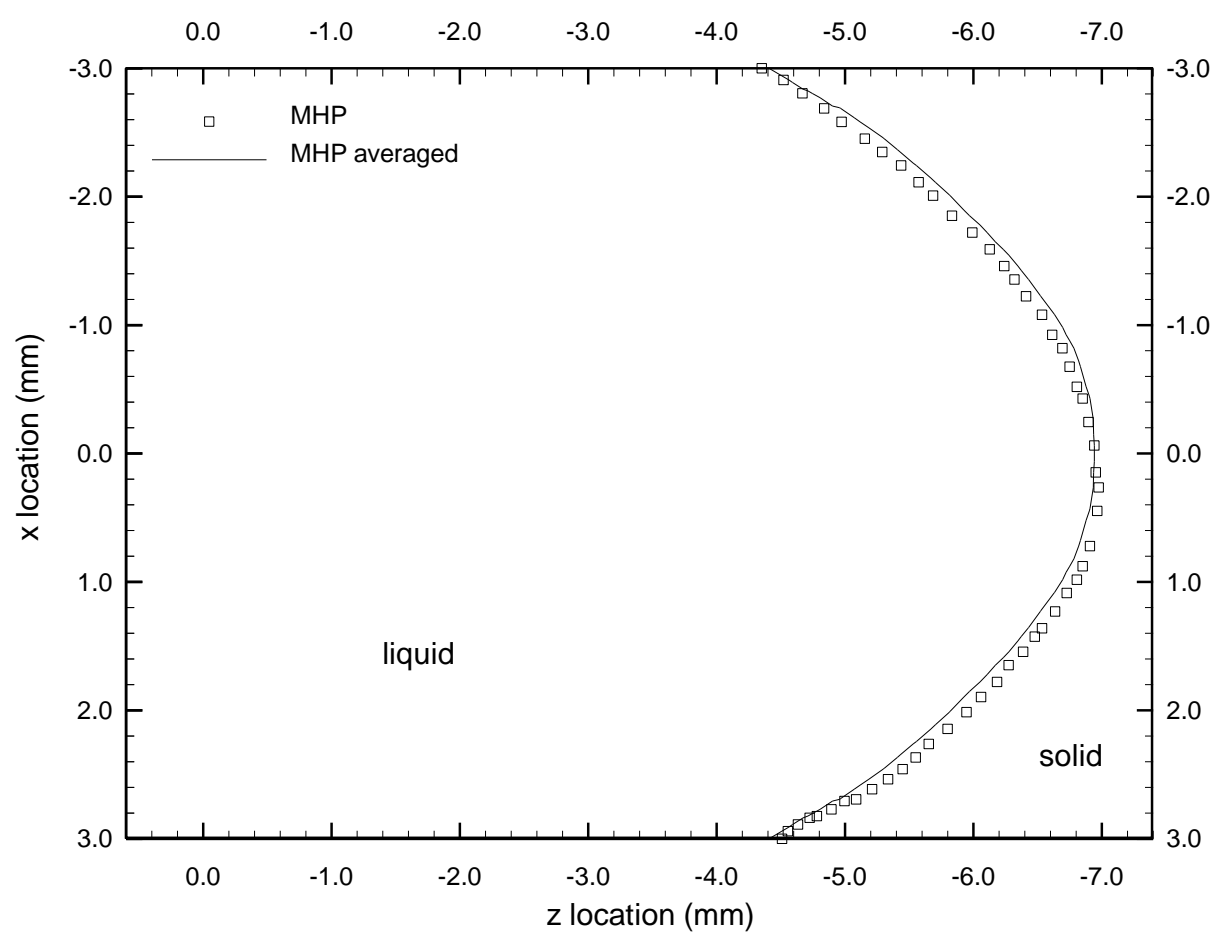

(a)

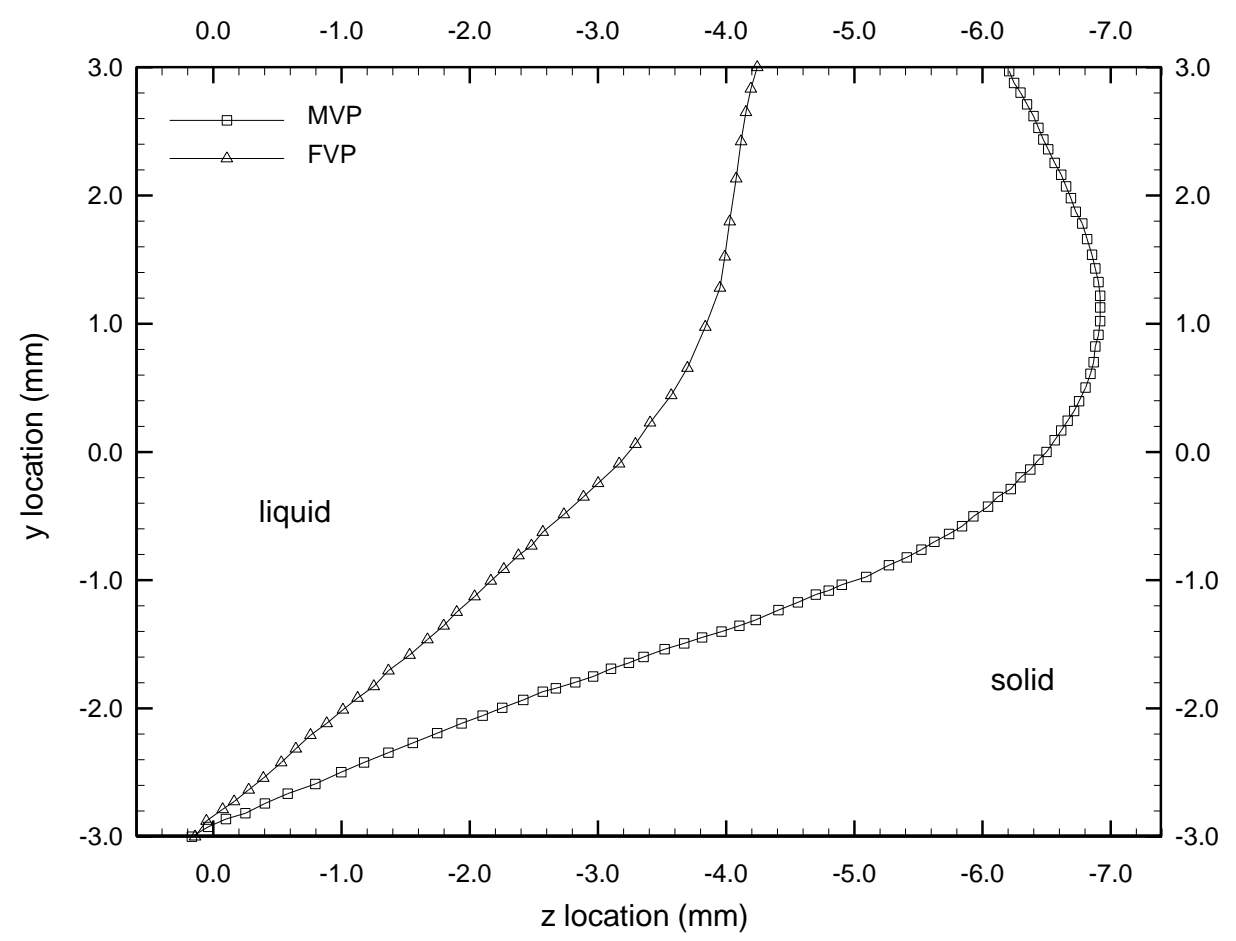

(b)

Fig. 8, Simpson et al. 


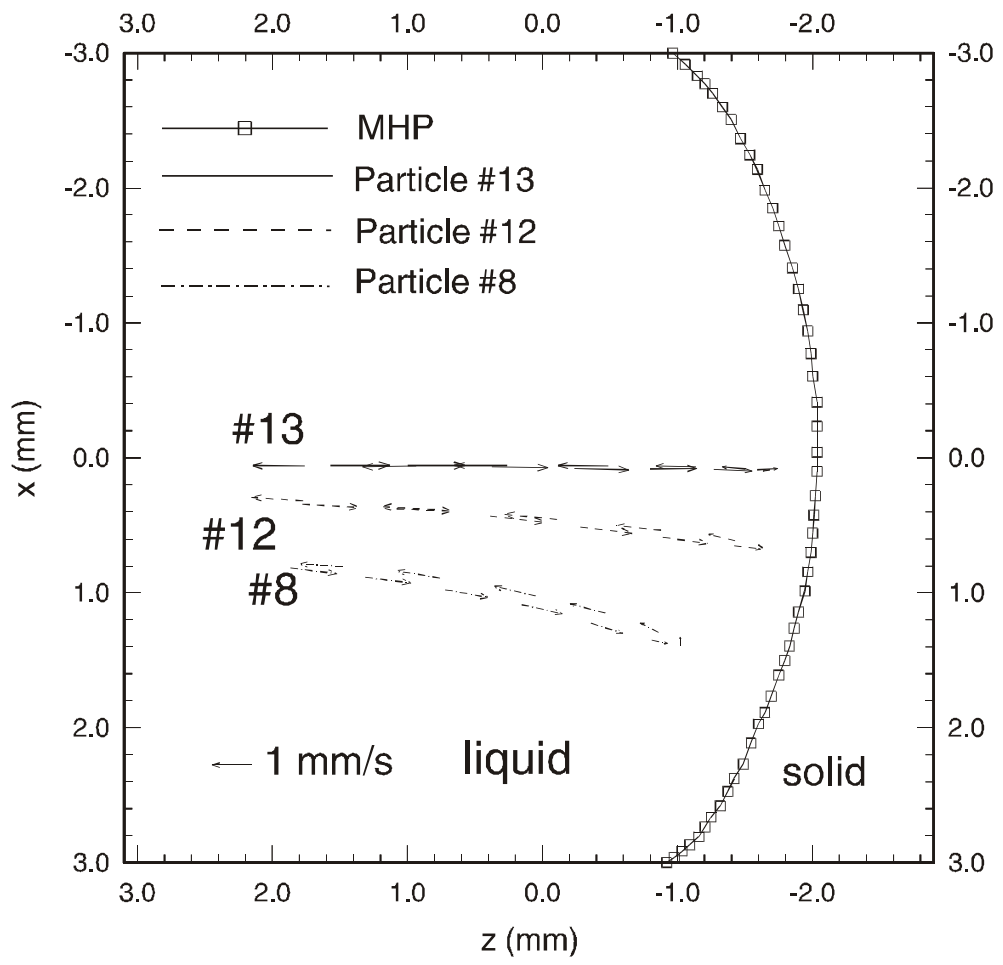

(a)

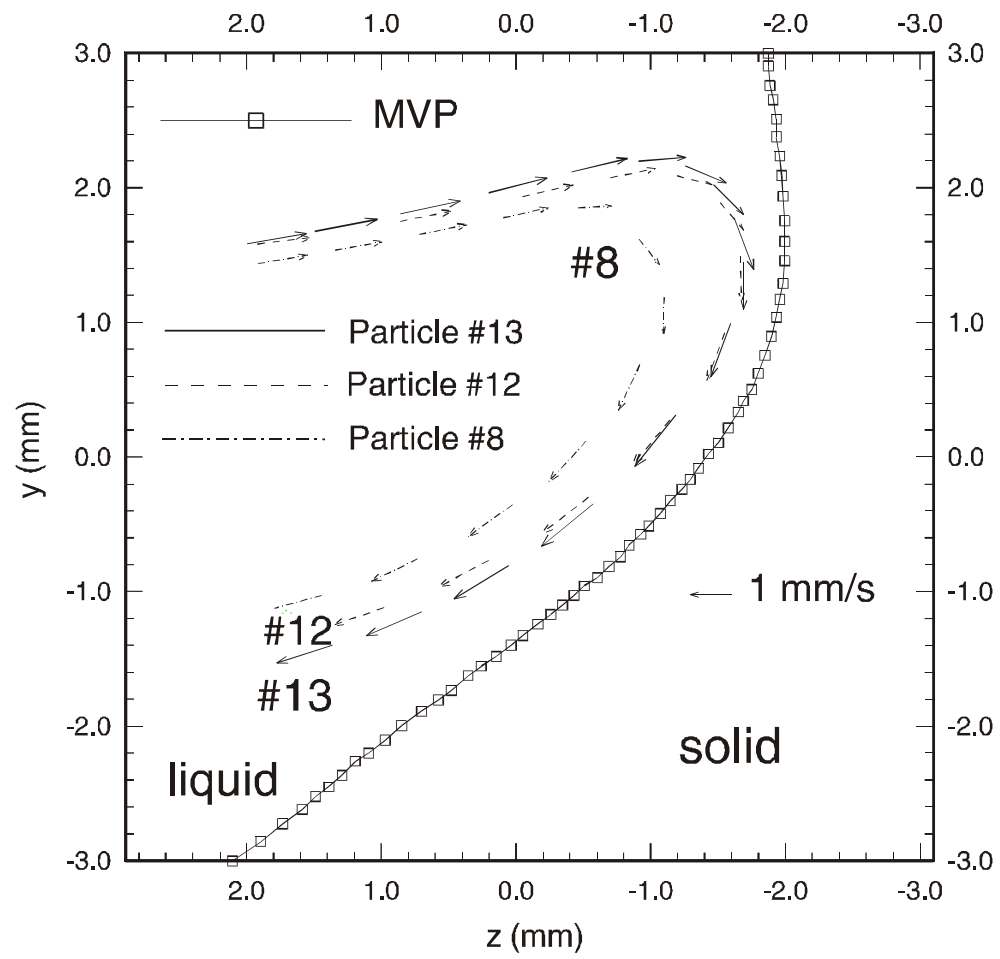

(b)

Fig. 9, Simpson et al. 

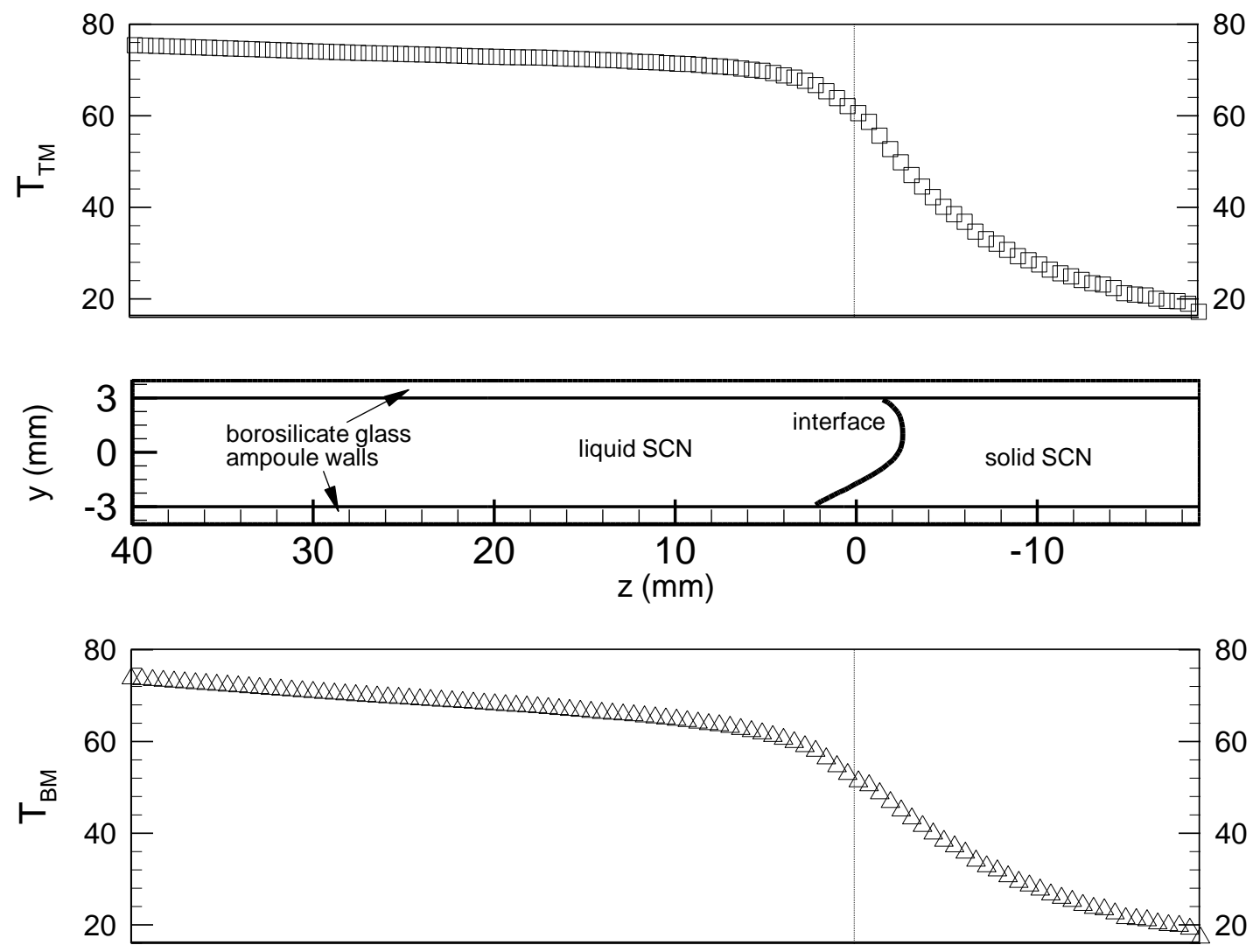

Fig. 10, Simpson et al. 
- $1.5 \mathrm{~mm} / \mathrm{s}$

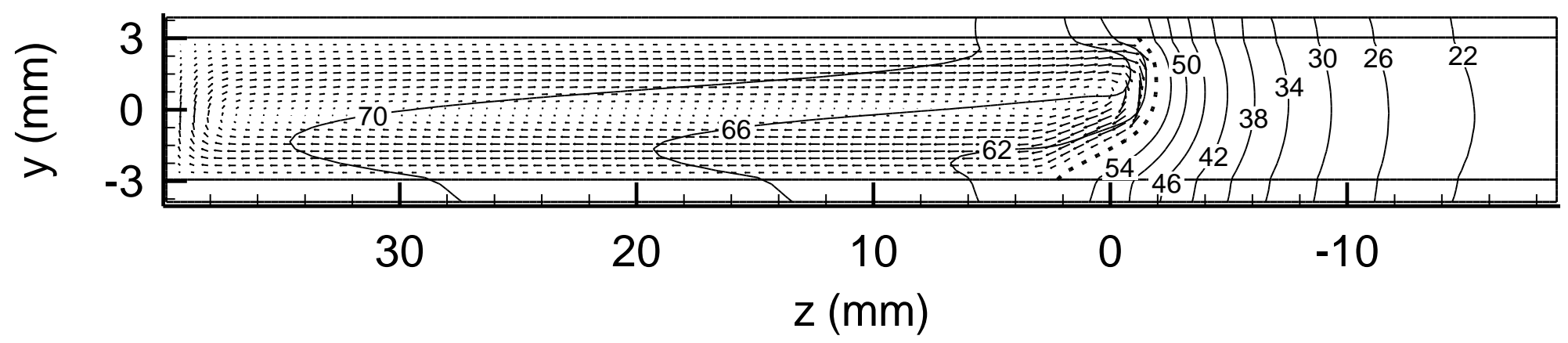

(a)

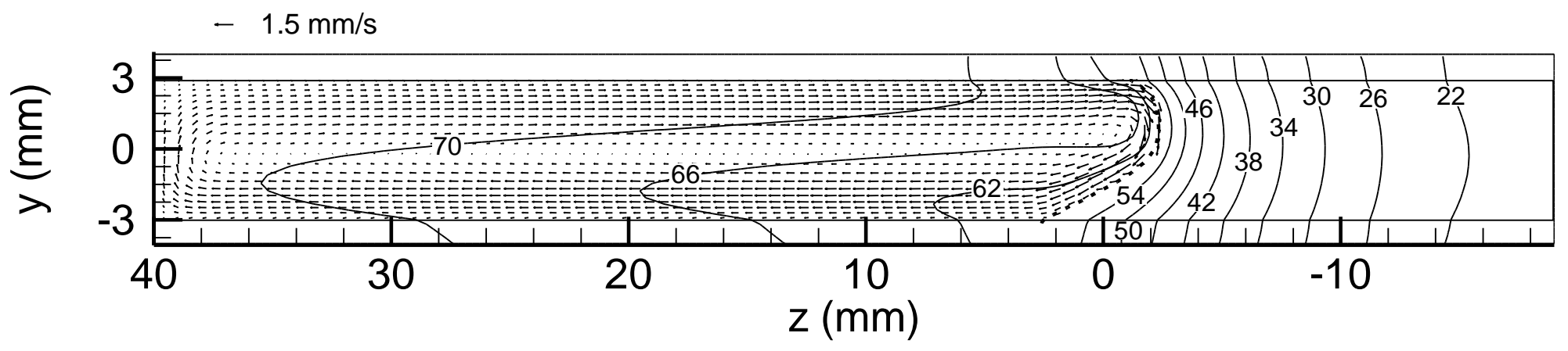

(b)

Fig. 11, Simpson et al. 


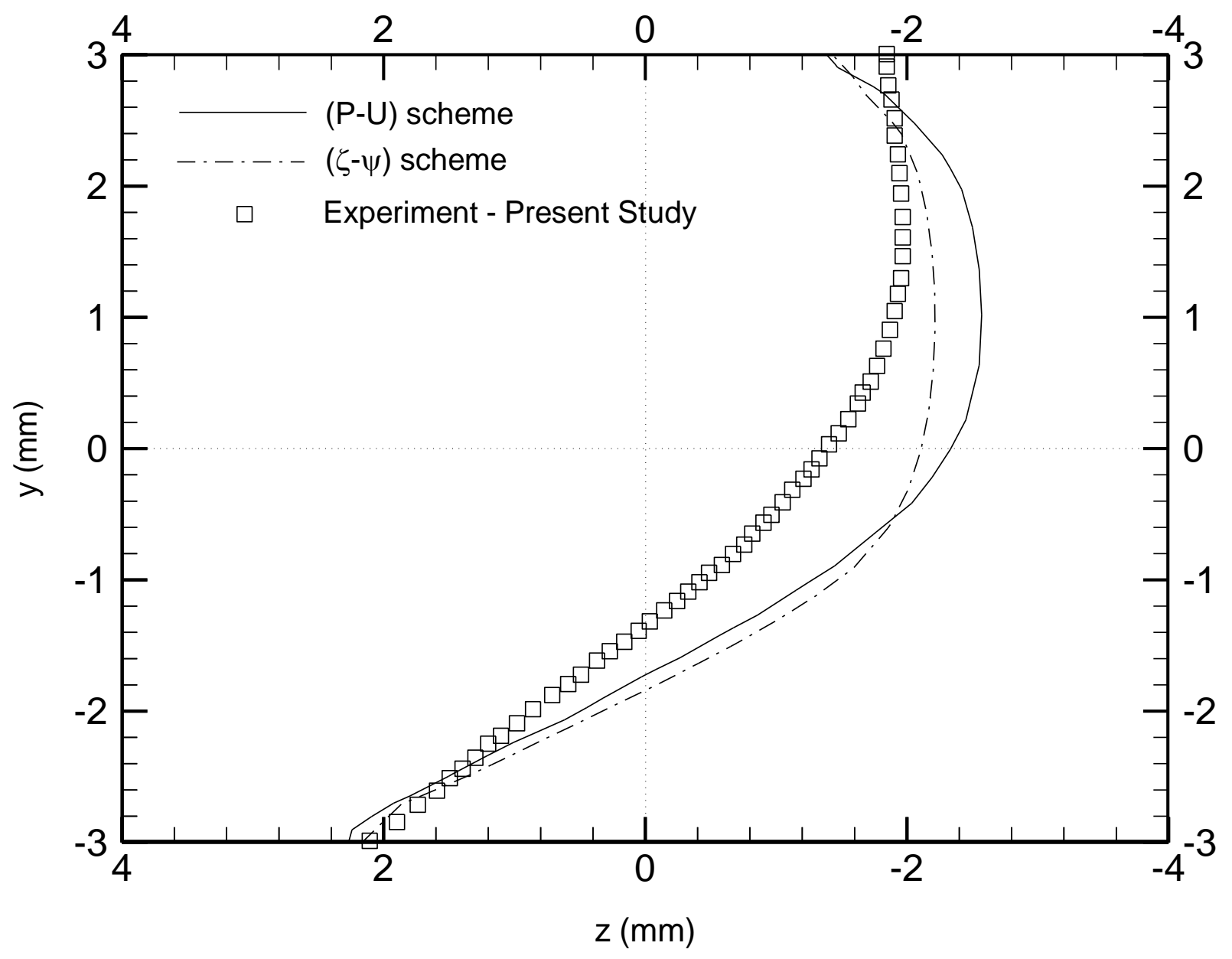

Fig. 12, Simpson et al. 

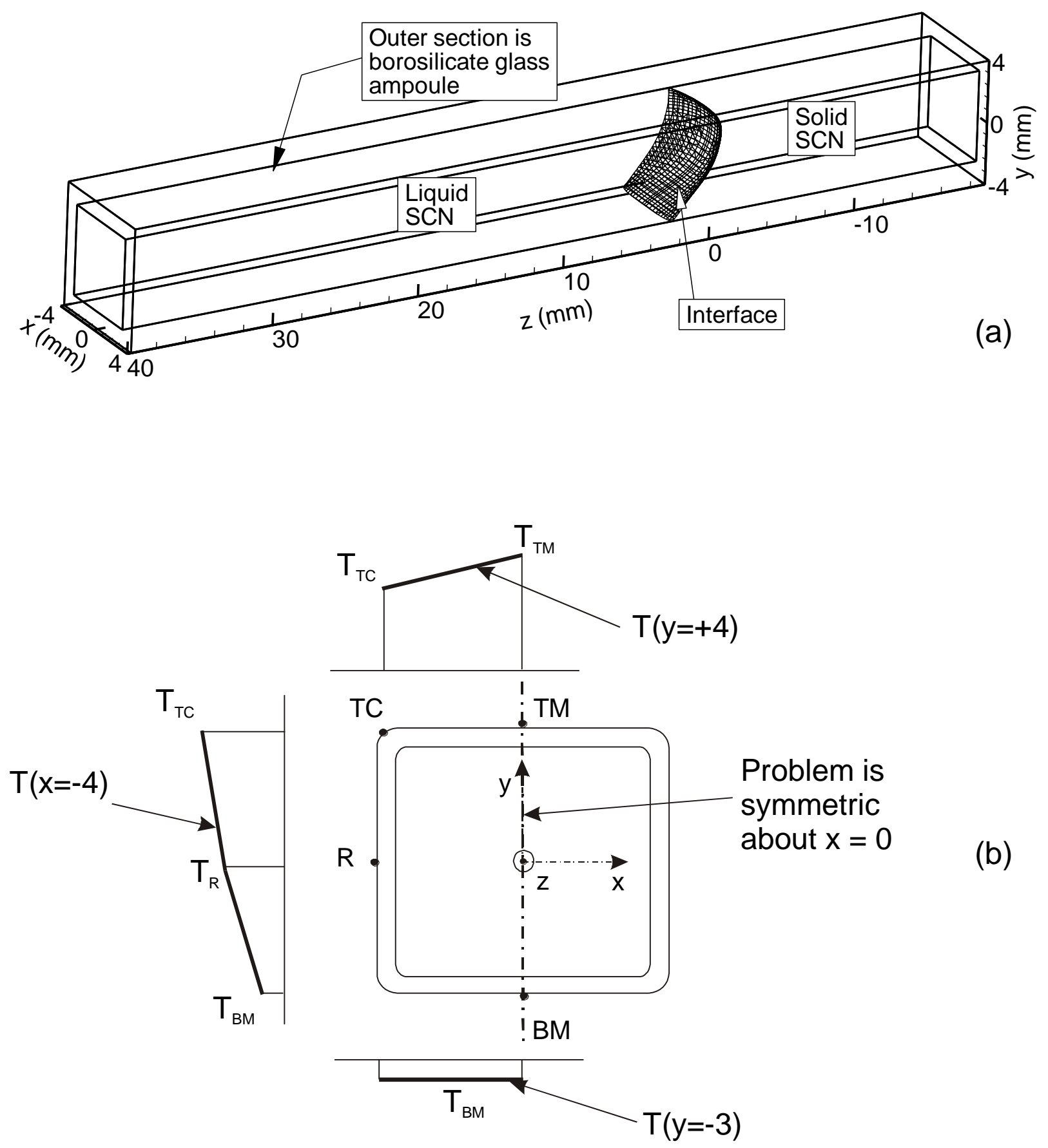

(b)

Fig. 13, Simpson et al. 


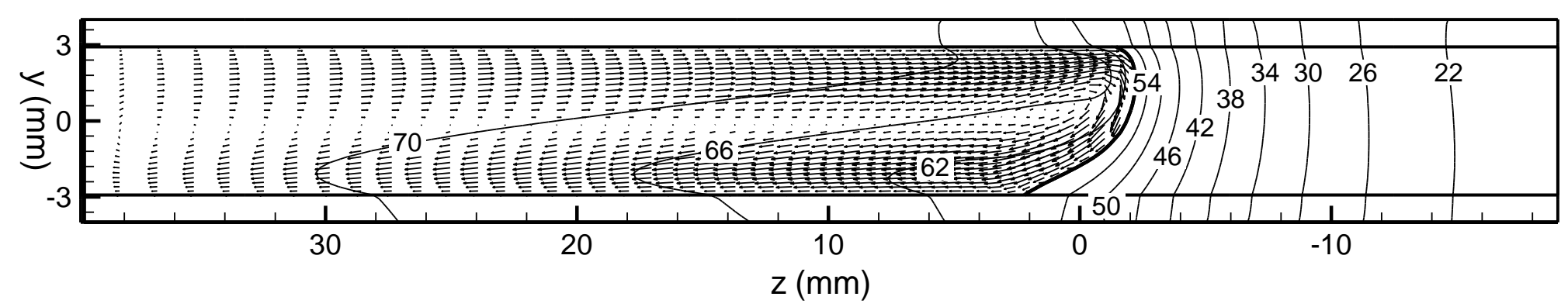

(a)

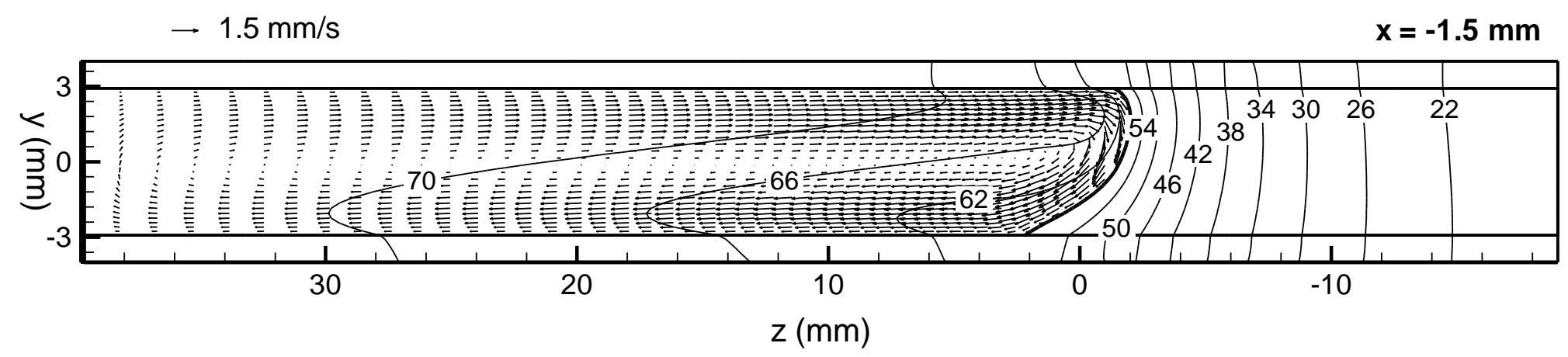

(b)

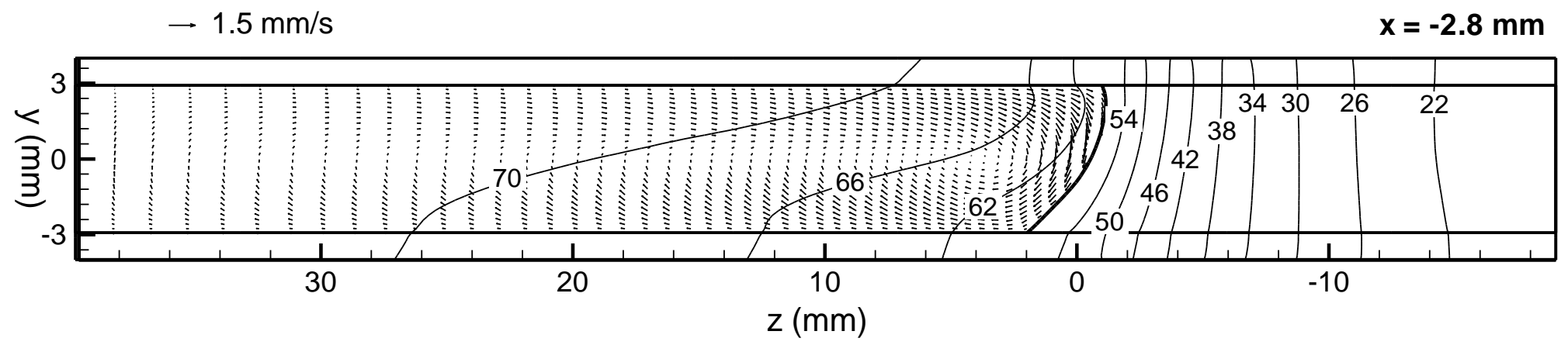

(c)

Fig. 14(a)-(c), Simpson et al. 


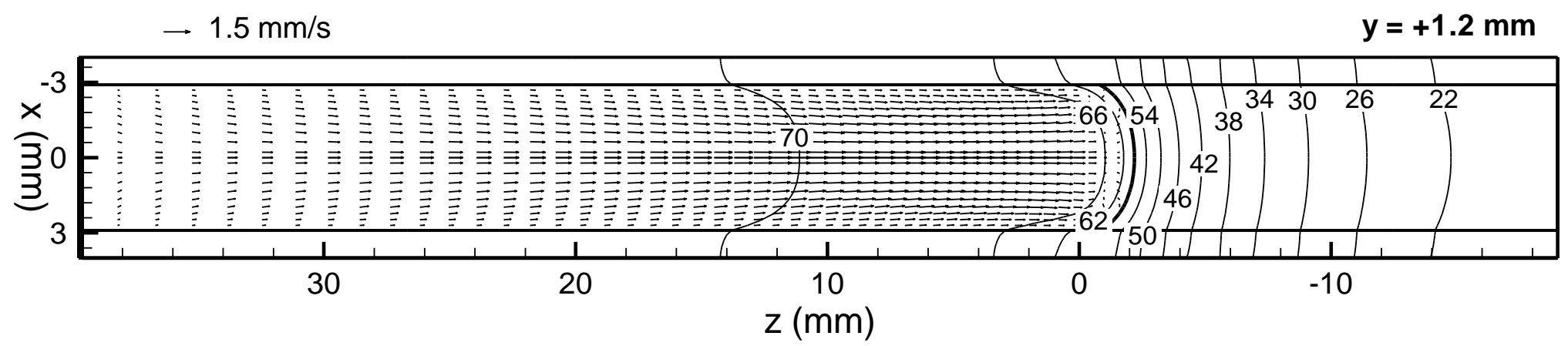

(d)

Fig. 14(d), Simpson et al. 


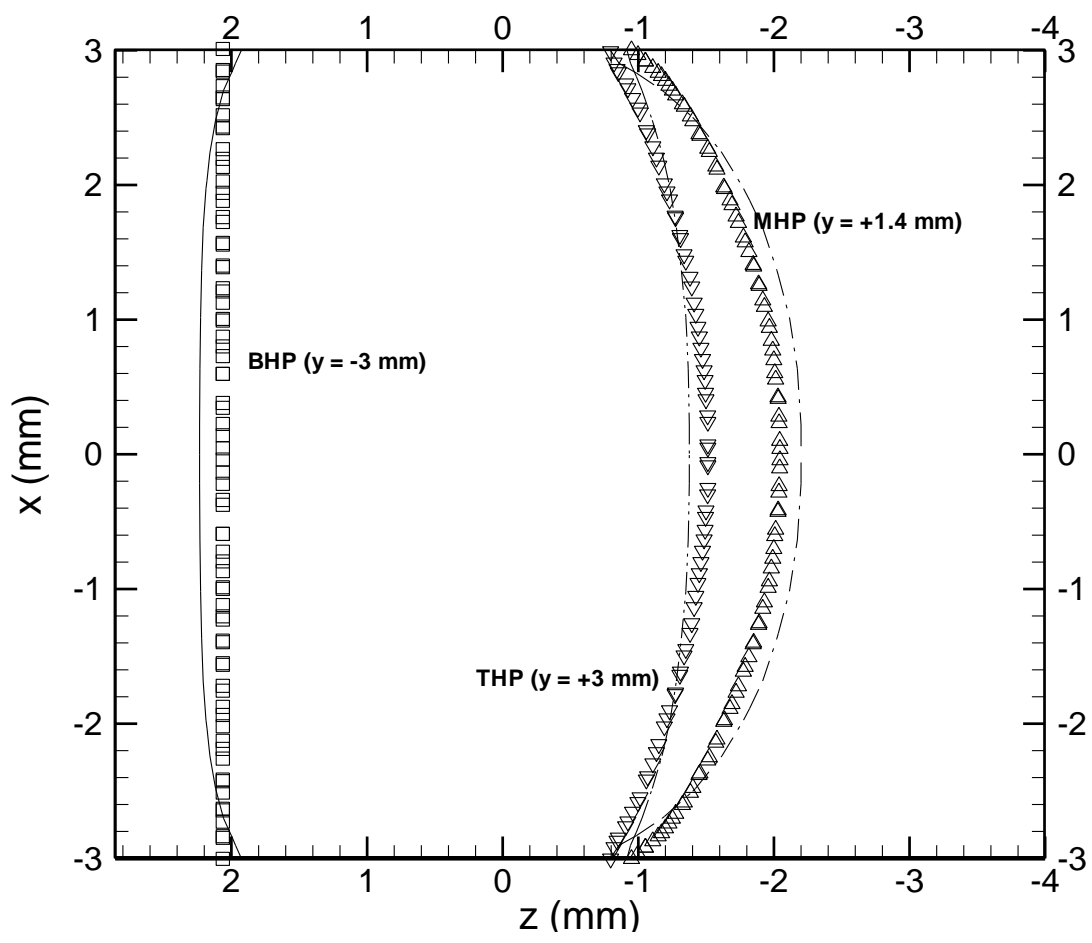

(a)

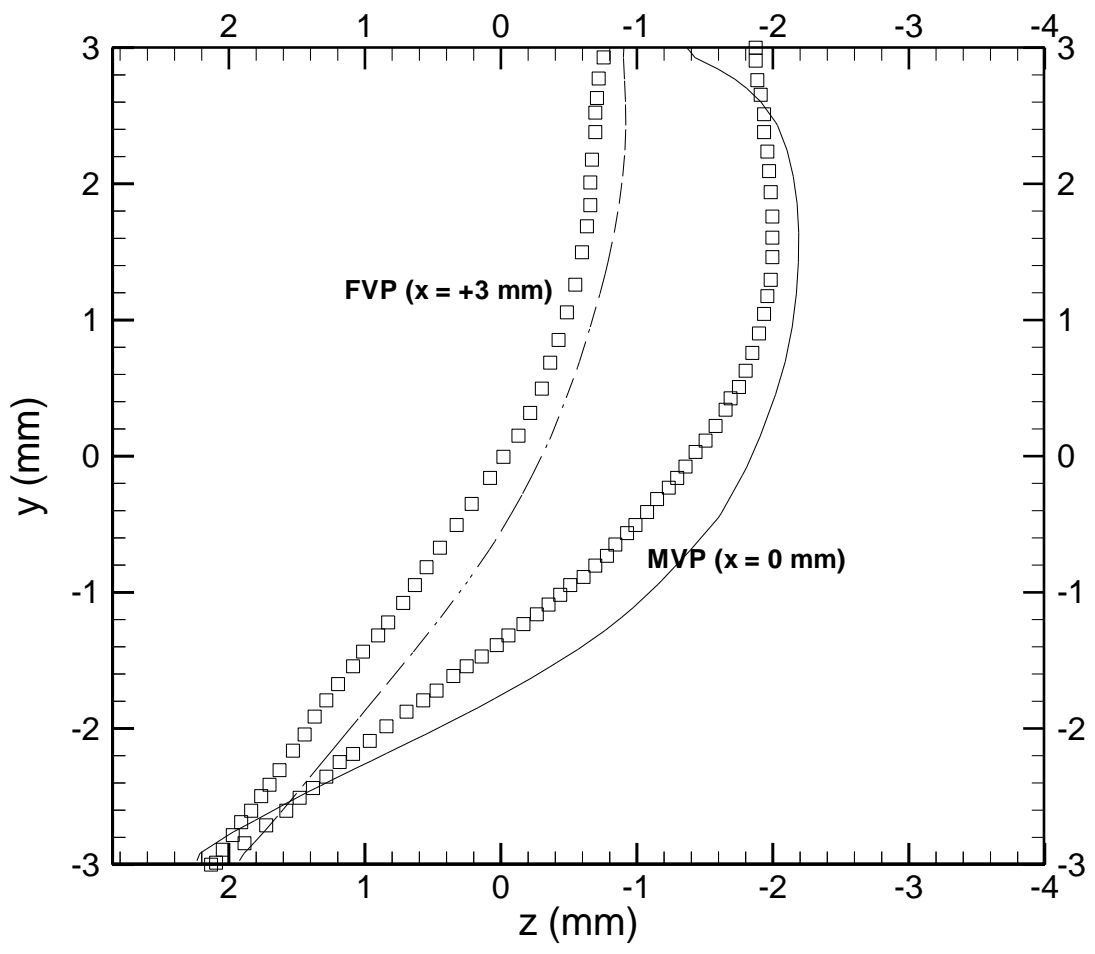

(b)

Fig. 15, Simpson et al. 


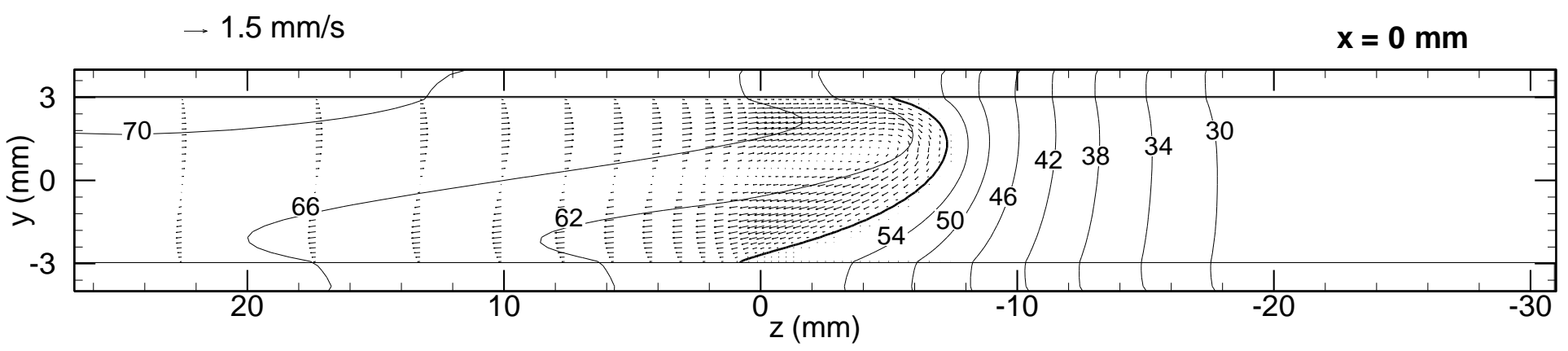

(a)

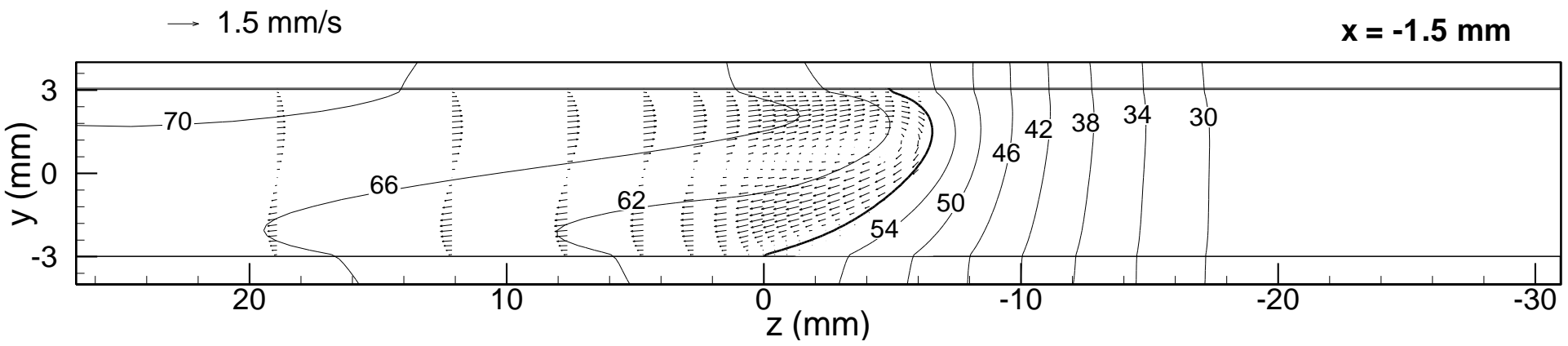

(b)

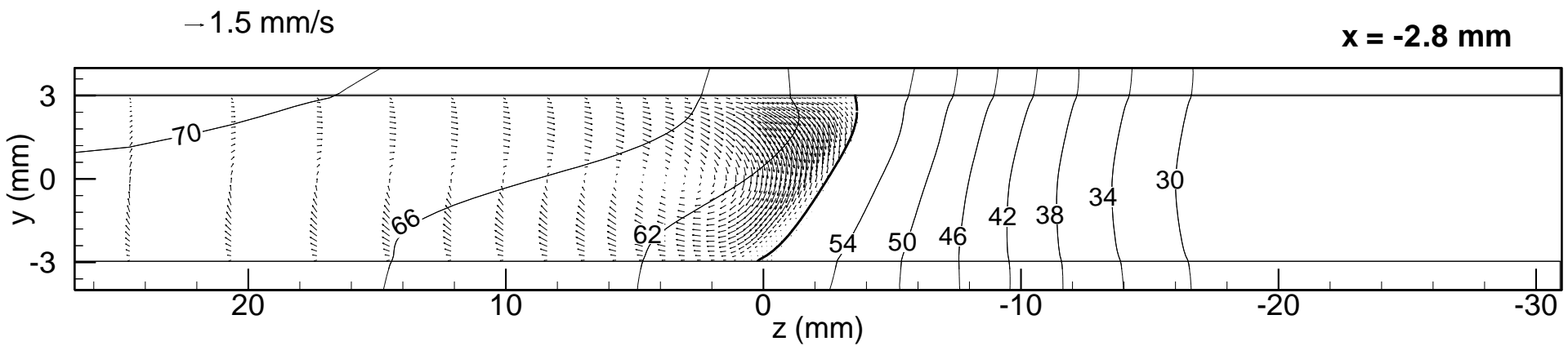

(c)

Fig. 16(a)-(c), Simpson et al. 


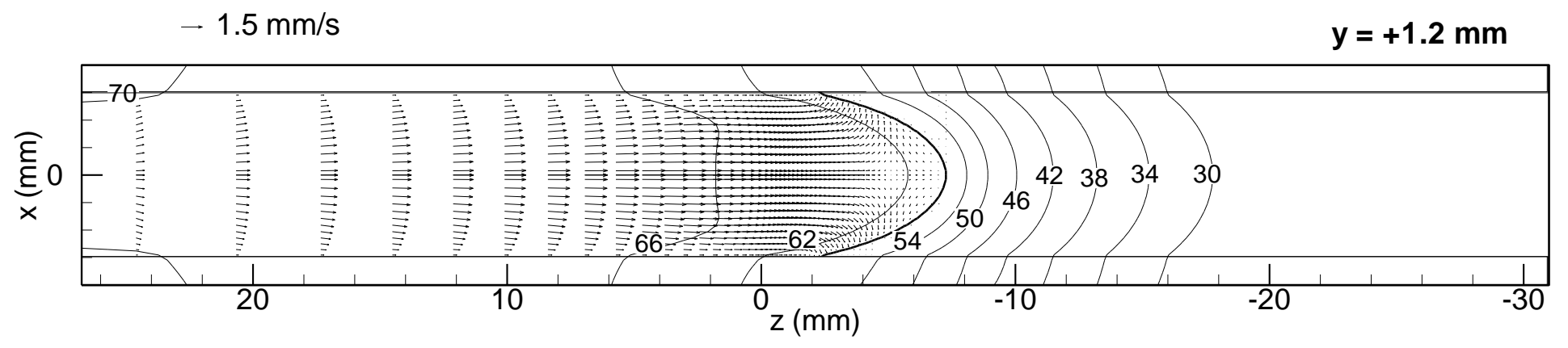

(d)

Fig. 16(d), Simpson et al. 


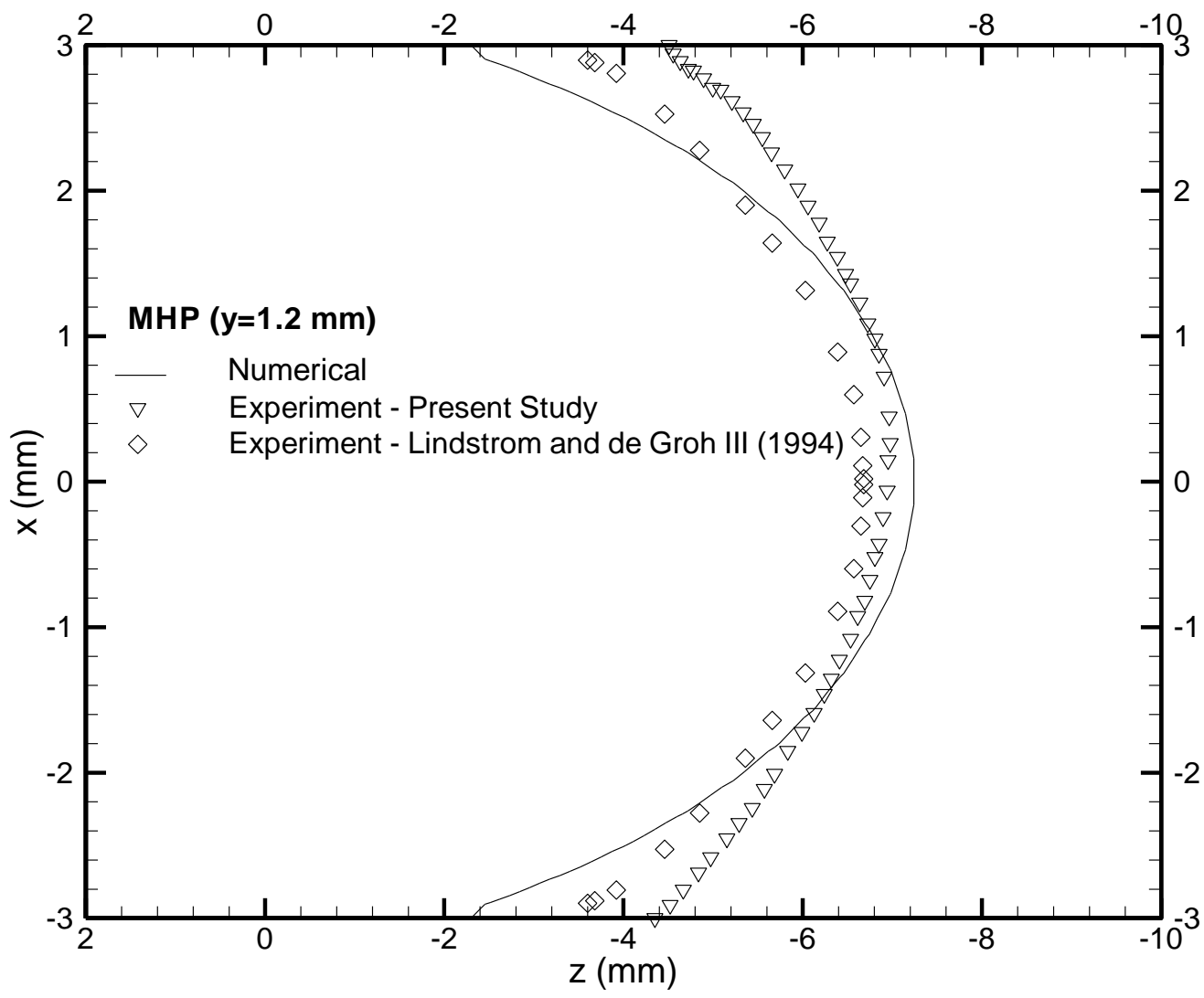

(a)

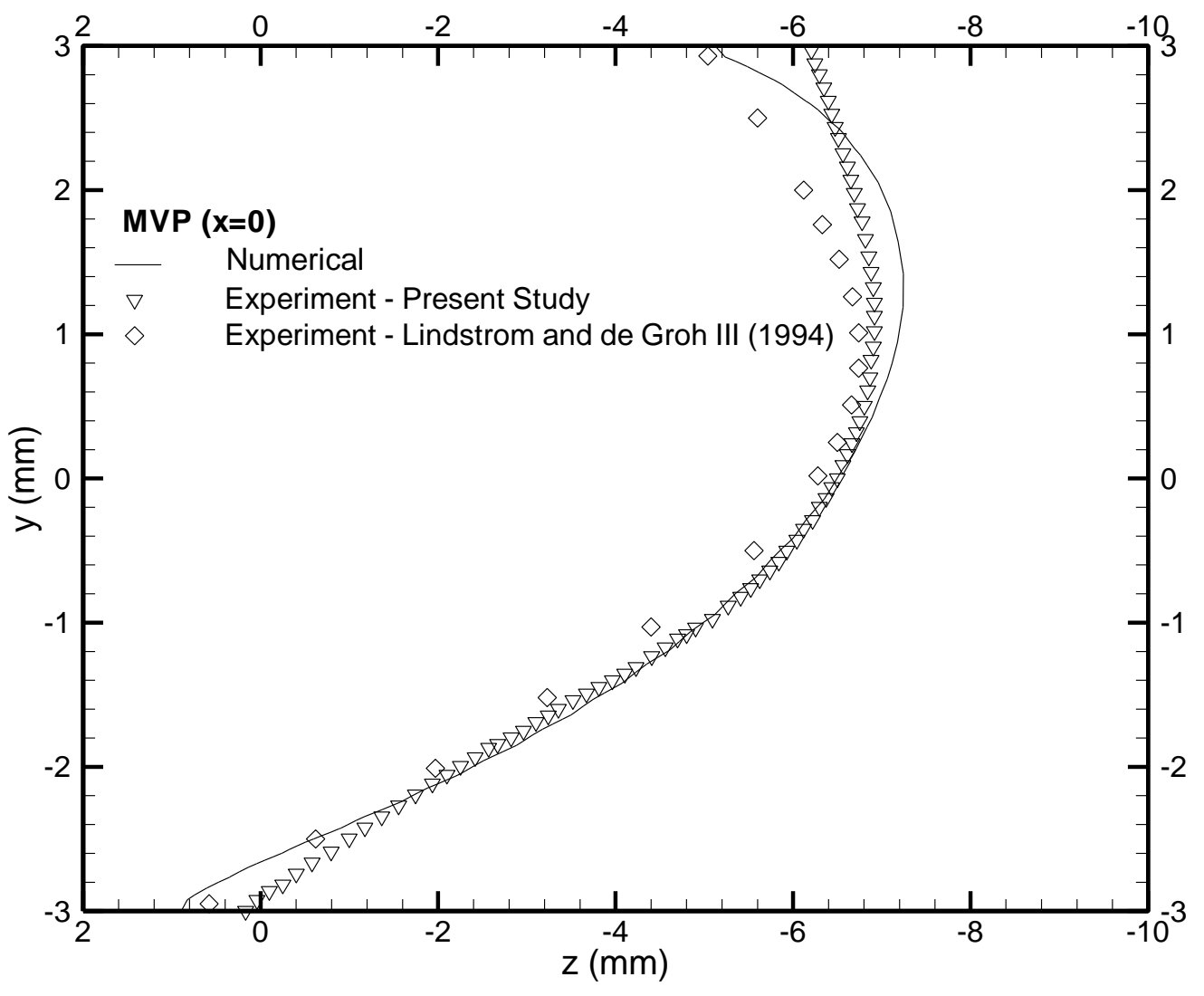

(b)

Fig. 17, Simpson et al. 